\title{
A spectral method for discriminating thermodynamic phase and retrieving cloud optical thickness and effective radius using transmitted solar radiance spectra
}

\author{
S. E. LeBlanc ${ }^{1,2, *}$, P. Pilewskie ${ }^{1,2}$, K. S. Schmidt ${ }^{2}$, and O. Coddington ${ }^{2}$ \\ ${ }^{1}$ University of Colorado, Department of Atmospheric and Oceanic Sciences, Boulder, CO, USA \\ ${ }^{2}$ University of Colorado, Laboratory for Atmospheric and Space Physics, Boulder, CO, USA \\ *now at: NASA Ames Research Center, Moffett Field, CA, USA
}

Correspondence to: S. E. LeBlanc (samuel.leblanc@nasa.gov)

Received: 21 March 2014 - Published in Atmos. Meas. Tech. Discuss.: 3 June 2014

Revised: 8 December 2014 - Accepted: 16 February 2015 - Published: 20 March 2015

\begin{abstract}
A new retrieval scheme for cloud optical thickness, effective radius, and thermodynamic phase was developed for ground-based measurements of cloud shortwave solar spectral transmittance. Fifteen parameters were derived to quantify spectral variations in shortwave transmittance due to absorption and scattering of liquid water and ice clouds, manifested by shifts in spectral slopes, curvatures, maxima, and minima. To retrieve cloud optical thickness and effective particle radius, a weighted least square fit that matched the modeled parameters was applied. The measurements for this analysis were made with the ground-based Solar Spectral Flux Radiometer in Boulder, Colorado, between May 2012 and January 2013. We compared the cloud optical thickness and effective radius from the new retrieval to two other retrieval methods. By using multiple spectral features, we find a closer fit (with a root mean square difference over the entire spectra of $3.1 \%$ for a liquid water cloud and $5.9 \%$ for an ice cloud) between measured and modeled spectra compared to two other retrieval methods which diverge by a root mean square of up to $6.4 \%$ for a liquid water cloud and $22.5 \%$ for an ice cloud. The new retrieval introduced here has an average uncertainty in effective radius $( \pm 1.2 \mu \mathrm{m})$ smaller by factor of at least 2.5 than two other methods when applied to an ice cloud.
\end{abstract}

\section{Introduction}

Clouds strongly influence Earth's radiative energy balance by modulating the transfer of solar radiation through the atmosphere. Clouds reduce the globally and annually averaged solar radiation absorbed at the surface by $53 \mathrm{~W} \mathrm{~m}^{-2}$ and produce a net cooling of $21 \mathrm{~W} \mathrm{~m}^{-2}$ (Allan, 2011). Cloud radiative effects are governed by optical thickness $(\tau)$, cloud particle effective radius $\left(r_{\mathrm{e}}\right)$, and thermodynamic phase $(\phi)$ (see, for example, Key and Intrieri, 2000; Sun and Shine, 1995; Wiscombe et al., 1984). Droplet size and water phase influence single scattering and absorption, primarily in the nearinfrared (NIR) region (Slingo, 1990; Twomey and Bohren, 1980; Wiscombe et al., 1984). For example, a liquid water droplet scatters more strongly in the forward direction than an ice particle of equivalent size (e.g., Baum et al., 2011). Furthermore, differences in bulk liquid water and ice absorption in the NIR (between 700 and $2500 \mathrm{~nm}$ ) have observable consequences in cloud spectral reflectance and transmittance that can be exploited to retrieve cloud thermodynamic phase (e.g., Pilewskie and Twomey, 1987).

In the spectral region emitted by the sun, optical thickness, particle size, and thermodynamic phase are most often retrieved using reflectance measurements (e.g., Nakajima and King, 1990; Platnick et al., 2001; Twomey and Cocks, 1989). Radiation reflected at cloud top has been scattered by particles in the uppermost regions of clouds, unlike transmitted radiation which has interacted with particles throughout the entire cloud layer. For this reason, transmittance-based 
retrievals are more representative of layer-average properties (Platnick, 2000) than their reflectance-based counterparts.

Uncertainties in retrieved cloud optical thickness and effective radius from transmittance are often much larger than from reflectance, particularly for thin clouds (Turner et al., 2007). Large uncertainties arise in transmittance retrievals because the information content from inverse methods optimized for reflectance is reduced when applied to cloud transmittance, particularly for particle size. This demands new methods of extracting information based on the unique physics of cloud transmittance, which is revealed in the observed spectral signatures. Another problem with transmittance-based retrievals is that optical thickness is not uniquely defined for all given transmittance, requiring additional information to retrieve optical thickness, such as if the cloud is optically thin (smaller than 4) or optically thick (larger than 4).

These problems have motivated the development of novel retrieval approaches specific to cloud transmittance. Such approaches include the transmittance at two wavelengths for a cloud overlying a vegetated surface (Chiu et al., 2010; Marshak et al., 2004), the transmittance at two wavelengths where condensed water absorption varies (Kikuchi et al., 2006; Rawlins and Foot, 1990), differential optical absorption spectroscopy (DOAS) (Daniel, 2002; Daniel et al., 2003, 2006; Schofield et al., 2007), and the slope of transmittance in selected spectral bands (McBride et al., 2012, 2011). Each of these methods has limitations. For example, using transmittance at wavelengths of differing condensed water absorption (Kikuchi et al., 2006; Rawlins and Foot, 1990) results in non-unique solutions of $\tau$ and $r_{\mathrm{e}}$ at optical thicknesses below 10 and large uncertainties in $r_{\mathrm{e}}$ at larger optical thicknesses. DOAS requires either assumptions or measurements of the photon path length to retrieve $\tau, r_{\mathrm{e}}$, and $\phi$. The method described by McBride et al. (2011) has higher sensitivity to effective radius than others, yet it is susceptible to systematic biases in absolute radiometric calibration and surface albedo (Coddington et al., 2013) and has thus far been applied to liquid clouds only.

The current work introduces 15 parameters that quantify several spectral features of normalized cloud-transmitted radiance that are sensitive to $\tau, r_{\mathrm{e}}$, and $\phi$. This work is motivated by McBride et al. (2011) who derived one parameter: the spectral slope of transmittance between 1565 and $1637 \mathrm{~nm}$ to retrieved liquid cloud $\tau$ and $r_{\mathrm{e}}$. In this work, a wide-ranging spectral method is derived that incorporates characteristics of the transmittance spectra that have not been previously exploited in a retrieval scheme. Cloud-transmitted radiance, or transmittance, is an inherent property of the cloud and is quantified by below-cloud zenith radiance measurements. Furthermore, the sensitivity of the 15 parameters to instrument calibration biases is reduced by utilizing normalized radiance. To find the most likely combination of $\tau$, $r_{\mathrm{e}}$, and $\phi$ we implement a two-step least-squares fit of measured parameters to modeled parameters. Thermodynamic phase is retrieved first, followed by $\tau$ and $r_{\mathrm{e}}$. In this work, clouds are assumed to be either composed of pure ice or pure liquid water and not a combination thereof. A quantitative analysis of the retrieval scheme using the GEneralized Nonlinear Retrieval Analysis (GENRA; Vukicevic et al., 2010) will be presented in a future work which will include an uncertainty and sensitivity analysis of ancillary inputs.

Section 2 details the instrumentation and measurements used in this work. Section 3 details the radiative transfer model, the variance in radiance spectra due to changes in optical thickness, effective radius, and thermodynamic phase, and the 15 parameters. In Sect. 4, we introduce the retrieval and analysis methods and apply the retrieval to measured radiance spectra and compare the results to other retrieval methods. Section 5 summarizes this work and presents its important conclusions. Extra details of the case studies are presented in Appendix A.

\section{Instrumentation and measurements}

Measurements of the spectral signatures of cloud transmittance were taken with the Solar Spectral Flux Radiometer (SSFR; McBride et al., 2011; Pilewskie et al., 2003). The SSFR is typically used to measure upwelling and downwelling spectral irradiance onboard airborne research platforms (e.g., Kindel et al., 2010; LeBlanc et al., 2012). In this study, the SSFR was deployed with a suite of instruments on a rooftop observatory (Skywatch, http://skywatch.colorado. edu/) located above the Duane Physical Laboratory at the University of Colorado, Boulder (see Sect. 2.2).

\subsection{Solar spectral flux radiometer}

The ground-based SSFR used in this study has a spectral resolution of $8-12 \mathrm{~nm}$ and covers the wavelength range between 350 and $1700 \mathrm{~nm}$ (McBride et al., 2011). SSFR has two optical inlets: one for measuring spectral downwelling irradiance and the other for spectral zenith radiance. The radiance light collector is composed of a collimating lens with a set of baffles to restrict stray light from outside the narrow field-ofview of $2.8^{\circ}$. Stray light rejection was determined by rotating the light collector with respect to a collimated stable light source at $50 \mathrm{~cm}$. Stray light was rejected by a factor of $10^{-5}$, below the noise floor of the SSFR (0.1-0.2\%; Pilewskie et al., 2003). The radiance calibration was performed with a NIST-traceable lamp illuminating a flat Spectralon panel of known reflectance. The flat panel was viewed with the radiance light collector at $45^{\circ}$ from normal to the panel.

The accuracy of SSFR, determined using a NIST-traceable lamp, is $3-5 \%$ over the spectral range of SSFR. The SSFR was calibrated at a calibration facility below the measurement site three times over the course of the multi-month measurement period, effectively bracketing the deployment period. A change of $8 \%$ was observed in successive absolute 
radiometric calibrations. Because of the large time gap between calibrations, rather than correct the data using updated calibrations, which would have additional uncertainties due to interpolation error, we estimated absolute accuracy to be $8 \%$, the conservative upper limit based upon the spread in calibration curves. The spectrum-to-spectrum average variation, or precision, was much better at $0.01 \mathrm{~W} \mathrm{~m}^{-2} \mathrm{~nm}^{-1} \mathrm{sr}^{-1}$ $(0.2 \%)$ for a midvisible wavelength (near $500 \mathrm{~nm})$, determined by the standard deviation of measurements from a stable light source.

When a radiance spectrum is normalized by its maximum value (found to always lie between 451 and $490 \mathrm{~nm}$ ) or by its value at $1000 \mathrm{~nm}$, accuracy depends on wavelength-towavelength stability of the sensor. This stability is evaluated by the relative change of each wavelength in the three radiometric calibrations. The mean variation between radiance evaluated at each wavelength and the maximum radiance was $4.6 \%$ (or $1.1 \%$ for normalizing by the radiance at $1000 \mathrm{~nm}$ ). This represents less variation throughout the measurement period than the absolute radiometric accuracy. Finally, the instrument was spectrally calibrated by measuring the output from a HeNe laser, Hg lamp, and a NIR laser diode.

\subsection{Skywatch observatory}

The Skywatch observatory (http://skywatch.colorado.edu/) hosts a number of instruments for measuring atmospheric radiation and precipitation. The instruments that provided data for this study are the pyranometer, pyrgeometer, ceilometer, micro rain radar (MRR), sky webcam, and spectral sunphotometer. The pyranometer and pyrgeometer measure broad band downwelling irradiance in the wavelength ranges of 300 to $2800 \mathrm{~nm}$ and 4500 to $42000 \mathrm{~nm}$, respectively. The ceilometer returns cloud base height by using a pulse diode laser lidar system at $910 \mathrm{~nm}$. The MRR is a K-band (24 GHz) vertical profiler of hydrometeors. The sunphotometer is a spectrometer system that tracks the sun and measures direct sunlight in the wavelength range of 350-1050 nm. These calibrated instruments provide ancillary measurements for estimating the cloud thermodynamic phase and evaluating the cloud homogeneity during SSFR measurement periods.

\subsection{Satellite measurements}

Coincident cloud observations were made with the Geostationary Operational Environmental Satellite (GOES, Minnis et al., 1995) and the Moderate Resolution Imaging Spectroradiometer (MODIS, Platnick et al., 2003). For selected cases studied in this work, cloud top height from GOES was available for 25 May 2012 and 6 August 2012, with a pixel size of approximately $6(\mathrm{~km})$ at cloud top height. MODIS-derived cloud top height, cloud optical thickness, and effective radius were available on 10 January 2013, with a pixel size of $500(\mathrm{~m})$. We used the MODIS 16-day averages of sur- face albedo (Schaaf et al., 2002) for determining the surface albedo used in the radiative transfer modeling.

\section{Radiative transfer simulations and description of retrieval parameters}

\subsection{Radiative transfer model}

The radiative transfer model used in this study is the $\mathrm{N}$ stream DISORT 2.0 (Stamnes et al., 2000) with SBDART (Ricchiazzi et al., 1998) for atmospheric molecular absorption; both are publicly available within LibRadtran (Mayer and Kylling, 2005). We varied optical thickness from 1 to 100 in increments of 1 to 10 for thermodynamic phase and effective radius from 2.5 to $30 \mu \mathrm{m}$ in $2.5 \mu \mathrm{m}$ increments for liquid drops and from 10 to $60 \mu \mathrm{m}$ in $2.5 \mu \mathrm{m}$ increments for ice particles. For liquid water clouds, Mie scattering calculations were used to obtain the scattering phase function and single scattering albedo of the cloud droplets (Evans, 1998; Wiscombe, 1980). A gamma distribution with $\alpha=7$, typical for liquid clouds (Mayer and Kylling, 2005), was used to represent the size distribution of the cloud droplets for each value of effective radius. For ice crystals, we used the scattering models for the severely roughened general habit mixture described by Baum et al. (2011). The scattering phase function was represented by a set of 256 Legendre coefficients calculated using the method by $\mathrm{Hu}$ et al. (2000). We used 28 streams in DISORT 2.0 to model the spectral zenith radiance. The use of more Legendre coefficients than streams is accomplished by the truncation approximation developed by Nakajima and Tanaka (1988), which is incorporated in DISORT 2.0. The extraterrestrial solar spectral irradiance was taken from Kurucz (1994) at $1 \mathrm{~nm}$ spectral resolution and convolved to the slit function and spectral resolution of the SSFR.

\subsection{Ancillary inputs}

Spectral radiance transmitted through clouds was modeled using a set of ancillary inputs given by a prescribed spectral surface albedo, cloud base altitude, cloud extent, and atmospheric state. The atmospheric state is defined by the profiles of number concentration of atmospheric gases, pressure, temperature, and water vapor calculated from soundings taken at the Denver airport for each day of interest (see Appendix A). Even though $\tau, r_{\mathrm{e}}$, and $\phi$ control most of the variance in radiance, these sets of ancillary inputs impact the spectral radiance through multiple reflections of the belowcloud radiation.

The accuracy and precision of the retrieved cloud properties depend on an accurate knowledge of the surface albedo and atmospheric state for each measurement period. By choosing conditions that occurred for each day during the measurement, we seek to reduce this source of error. Of the 3 days selected for case study, 2 days were in the 
spring/summer period (25 May 2012, 6 August 2012) when surface albedo was dominated by vegetation. The remaining day (10 January 2013) was in winter with a fresh snow albedo (see Appendix A).

\subsection{Modeled lookup tables (LUT)}

A set of modeled radiance spectra for varying $\tau, r_{\mathrm{e}}$, and $\phi$ (presented above) is used to build a grid of spectral parameters (described in Sect. 3.5) commonly referred to as lookup tables. The modeled radiance spectra were interpolated to a finer grid in $\tau$ (resolution of 1) and $r_{\mathrm{e}}$ (resolution of $1 \mu \mathrm{m}$ ). For each of these interpolated radiance spectra, we calculate the 15 parameters. This LUT of parameters is calculated for each case and multiple solar zenith angles with resolution 0.05 in cosine of the solar zenith angle, ranging from 0.6 to 0.75 for case A, 0.6 to 0.7 for case B, and 0.4 to 0.45 for case $\mathrm{C}$.

\subsection{Variability in normalized radiance spectra due to $\tau, r_{\mathrm{e}}$, and $\phi-$ physical basis}

The spectral features in cloud transmittance that vary with bulk absorption from liquid and ice (Fig. 1a), and its asymmetry parameter spectrum (Fig. 1b) were chosen as the basis for deriving the 15 parameters. The bulk ice absorption spectrum has local maxima that are shifted to longer wavelengths than maxima in liquid water absorption, themselves shifted to longer wavelengths than the absorption maxima in water vapor (Fig. 1a). Cloud-transmitted radiation at absorption maxima (for gas, liquid, and ice) is reduced more than the radiation at the surrounding wavelengths. For absorption by cloud particle, this is also dependent on particle size. Further reduction occurs due to multiple scattering within the cloud, the amount of which depends upon cloud optical thickness. Asymmetry parameter is a measure of the ratio of forward-scattering to back-scattering. The asymmetry parameter for ice crystals is generally lower than for liquid droplets of equivalent effective radius (see Fig. 1b), meaning that radiance transmitted through an ice cloud is consistently lower than radiance transmitted through a liquid cloud with the same optical thickness.

By modulating scattering and absorption properties of cloud particles, combinations of $\tau, r_{\mathrm{e}}$, and $\phi$ result in unique cloud-transmitted radiance spectra (Fig. 1). In general, the largest variability throughout the entire wavelength range, outside of the gas absorption bands, is controlled by changes in $\tau$. By normalizing each radiance spectra by its maximum, the broad range in magnitude due to $\tau$ (considered to be the first-order dependence on $\tau$ ) evident in Fig. 2a is virtually eliminated in Fig. 2b. The broad range in magnitude in the NIR (wavelengths longer than $700 \mathrm{~nm}$ ) due to $\tau$ is also eliminated when normalizing each radiance spectrum by its value at $1000 \mathrm{~nm}$. An added benefit of using normalized radiance is the reduction in uncertainty from $8 \%$ for radiance to
$4.6 \%$ for its maximum-normalized counterpart. In addition, the change in magnitude of radiance spectra in the NIR due to variation in solar zenith angles up to $80^{\circ}$ is nearly eliminated by normalizing the radiance spectra (not shown).

After normalization, the first-order dependence of the radiance spectra on $\tau$ is removed, but variability in spectral radiance linked to spectral features that differ for $r_{\mathrm{e}}, \phi$, and $\tau$ remains. By normalizing the radiance spectra, spectral features and shapes are amplified while the large-scale variation in magnitude of the zenith radiance spectra is eliminated (see Fig. 2). In some cases, the remaining spectral features, particularly in the NIR, become more distinct for normalized radiance spectra than non-normalized radiance spectra. One such case occurs for an ice cloud of $\tau=10$ (dashed red line), which coincides in the NIR with a liquid cloud of $\tau=50$ (solid blue line) (Fig. 2a). However, once normalized, these two clouds can be distinguished by their NIR spectral features (Fig. 2b): location of the local maximum is at $1050 \mathrm{~nm}$ for the liquid cloud (solid blue line) and at $1000 \mathrm{~nm}$ for the ice clouds (dashed red line); lower magnitude in normalized radiance near $1200 \mathrm{~nm}$ for the liquid than ice cloud; and steeper spectral slope near $1600 \mathrm{~nm}$ for the liquid than ice cloud.

Not all $\tau$ dependence is eliminated by normalization, especially at wavelengths near the liquid and ice absorption maxima in the NIR (see Fig. 1a). The effects of absorption in the NIR are amplified with increases in $\tau$, illustrated by the large variability of normalized radiance in the NIR in Fig. 3. For clouds with $\tau>4$, the radiance in the NIR decreases with increasing $\tau$ and asymptotes to zero. The amount by which the NIR radiance is decreased is due to the absorption by cloud particles.

Not only does an increase in $\tau$ reduce the NIR-transmitted signal, differences in spectrally dependent absorption also result in features that become more distinct with increases in $\tau$ (Fig. 3). Transmittance is defined here as the transmitted radiance normalized by top-of-cloud spectral radiance; note that for our purposes, the features characterizing the transmitted spectra would not change for either transmittance or transmitted radiance normalized by the maximum signal but may differ in magnitude; therefore cloud-transmitted normalized radiance and transmittance are used interchangeably when describing physical processes. As examples of spectral features linked to differences in liquid and ice absorption, we examine three wavelength ranges: (1) 950-1100 nm, (2) 1150$1350 \mathrm{~nm}$, and (3) $1450-1650 \mathrm{~nm}$. The location of the peak radiance in region (1) shifts from 1000 to $1060 \mathrm{~nm}$ as $\tau$ increases for liquid clouds (Fig. 3a). In the same wavelength range, radiance transmitted through an ice cloud has a local radiance minimum that deepens with increases in $\tau$. Another spectral feature is observed in normalized radiance transmitted through ice clouds in the wavelength range (2) where radiance at wavelengths longer than $1200 \mathrm{~nm}$ is preferentially absorbed. As $\tau$ increases, the normalized radiance in region (2) changes from a convex shape with a local maximum 

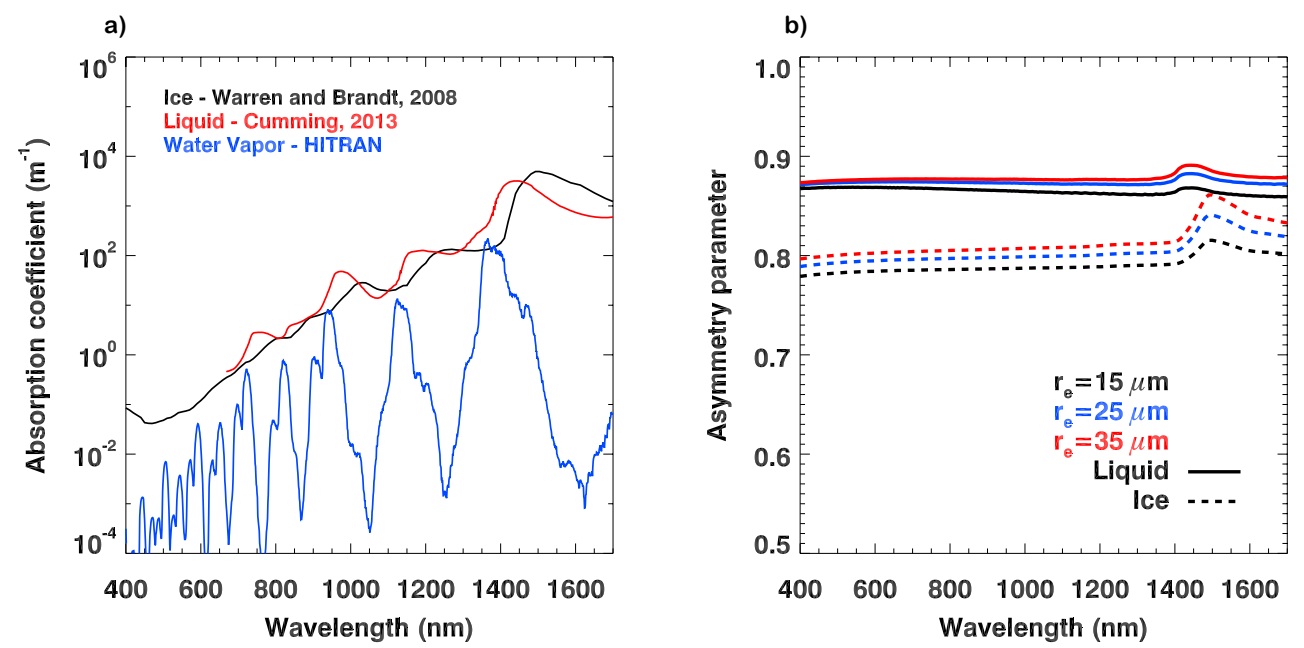

Figure 1. (a) Spectral bulk absorption coefficient and (b) asymmetry parameter for different thermodynamic phases of water. Notice the local maxima offset between ice water, liquid water, and water vapor near 900,1100 , and $1400 \mathrm{~nm}$. The bulk ice absorption coefficient has been determined by Warren and Brandt (2008), while the bulk liquid water absorption coefficient has been determined by Cumming (2013). The bulk water vapor absorption has been calculated using the HITRAN model described by Rothman et al. (2009) for an optical path length of $10 \mathrm{~m}$ with pure water vapor pressure of $15 \mathrm{hPa}$ at $293 \mathrm{~K}$ (Ptashnik et al., 2004). The asymmetry parameters for liquid drops were calculated from Mie scattering calculation following the methodology developed by Wiscombe (1980). Ice asymmetry parameter were obtained from the scattering models for severely roughened general habit mixture described by Baum et al. (2011).

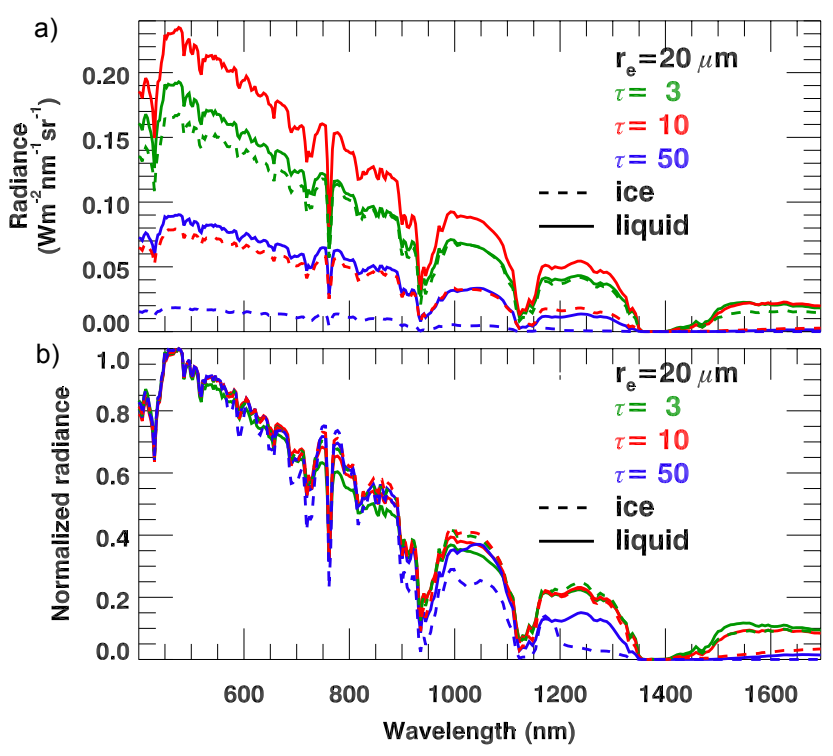

Figure 2. Modeled zenith radiance spectra transmitted through clouds with a few combinations of optical thickness $(\tau)$ for ice and liquid water clouds with an effective radius $\left(r_{\mathrm{e}}\right)$ of $20 \mu \mathrm{m}$ for the atmospheric state and surface albedo defined for 25 May 2012 and a solar zenith angle of $50^{\circ}$. Top panel (a) shows the radiance spectra in $\mathrm{W} \mathrm{m}^{-2} \mathrm{~nm}^{-1} \mathrm{sr}^{-1}$ units and bottom panel (b) shows the radiance spectra normalized by their maximum value (unitless). The radiance minima centered at 760, 940, 1130, and $1400 \mathrm{~nm}$ represent gas absorption bands that are ignored in this work. at $1230 \mathrm{~nm}$ to a concave shape with a local minimum. The spectral feature in range (3) is a curved shape with a maximum near $1500 \mathrm{~nm}$ that flattens with increases of $\tau$ for both ice and liquid clouds.

Since these spectral features are linked to absorption, whenever the transmittance is entirely attenuated these features disappear. This attenuation occurs at lower $\tau$ for ice clouds than liquid clouds in the wavelength range 1500 $1600 \mathrm{~nm}$ (see Fig. 3). This is caused by higher ice absorption than liquid in that wavelength range (see Fig. 1a). Transmittance through ice clouds at $1250 \mathrm{~nm}$ is also entirely attenuated at lower $\tau$ than at $1180 \mathrm{~nm}$ because of higher absorption at $1250 \mathrm{~nm}$ than $1180 \mathrm{~nm}$.

For clouds with $\tau<4$, where radiance in the mid-visible is still increasing with $\tau$, the normalized transmitted radiance spectra show an influence from molecular scattering. The spectra in Fig. 3a for $\tau=0.2$ matches more closely the clear sky spectra, which is inversely proportional to the fourth power of the wavelength, than the normalized radiance spectra for $\tau=100$, which is roughly proportional to the inverse of the wavelength. As $\tau$ is reduced, the magnitude of signal at wavelengths between 550 and $700 \mathrm{~nm}$ decreases and its slope becomes more negative until they match the spectrum of clear sky. The clear sky spectrum (green spectrum in Fig. 3a) is entirely dependent on scattering by molecules (Rayleigh scattering) and the solar zenith angle. The slope of the spectrum in the visible is proportional to $\tau$ until scattering by cloud particles dominates scattering by molecules. This transition occurs at lower $\tau$ for ice clouds (near 1) than liquid clouds (near 2), obscured by radiance transmitted through 


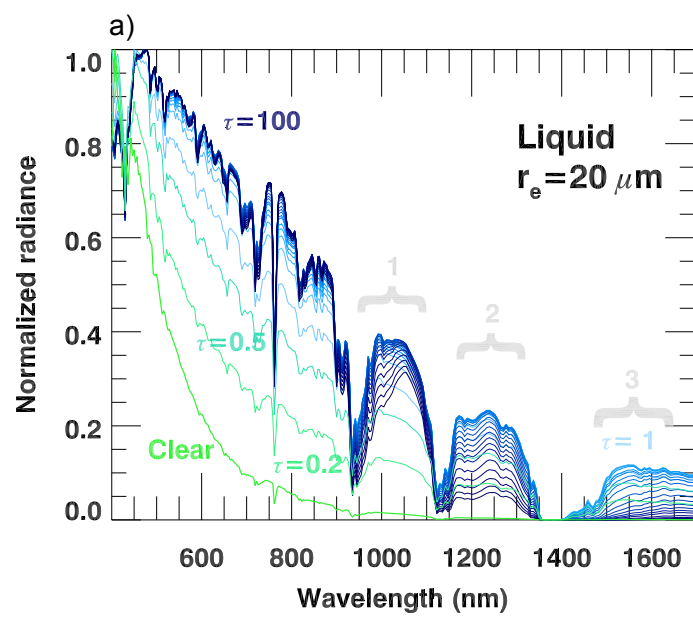

b)

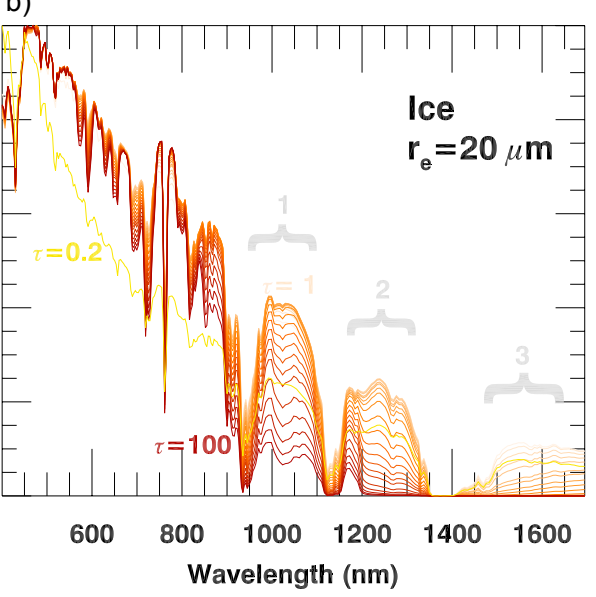

Figure 3. Normalized radiance spectra for liquid (left) and ice (right) clouds with an $r_{\mathrm{e}}$ of $20 \mu \mathrm{m}$ and $\tau$ varying from 0.2 to 100 (where darker colors denotes optically thicker clouds). A clear sky normalized radiance (denoted in green) is added for comparison to the optically thinnest clouds. Three wavelength ranges with spectral features are indicated by the grey curly brackets.

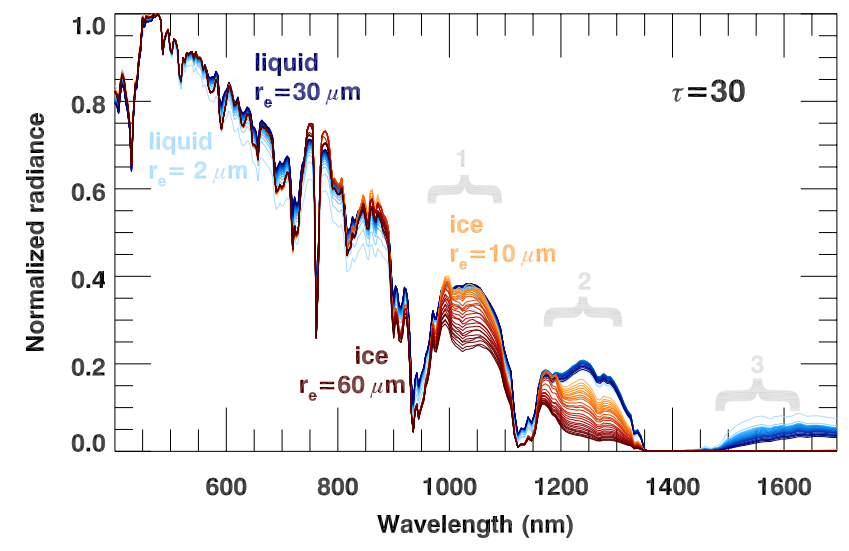

Figure 4. Normalized radiance spectra transmitted through liquid and ice clouds of $\tau$ of 30 and $r_{\mathrm{e}}$ ranging from 2 to $30 \mu \mathrm{m}$ for liquid clouds and 10 to $60 \mu \mathrm{m}$ for ice clouds. Darker orange lines represent radiance spectra transmitted through larger ice particles. Darker blue lines represent the radiance spectra transmitted through larger liquid cloud droplets. Three wavelength ranges with spectral features are indicated by the grey curly brackets.

optically thicker clouds in Fig. 3. After this transition, the slope of normalized radiance in the visible varies less and depends on $\tau, r_{\mathrm{e}}$, and $\phi$, rather than on molecular scattering. Similar results are also observed by Brückner et al. (2014), where instead of a slope in the mid-visible, they used a ratio of transmittance at 450 and $680 \mathrm{~nm}$.

The normalized spectra are modified by $\phi$ and $r_{\mathrm{e}}$, especially in the three wavelength ranges which show variations with $\tau$. Changes of normalized radiance due to $\phi$ and $r_{\mathrm{e}}$, when $\tau$ is kept constant, can be observed in Fig. 4. Outside of these three wavelength ranges and gas absorption bands, transmittance through both liquid and ice clouds overlaps for all cloud particle sizes except for particles smaller than $5 \mu \mathrm{m}$. In the three wavelength ranges, ice and liquid clouds can be distinguished by the shape and magnitude of the transmittance. Transmitted normalized radiance for ice clouds has the largest dynamic range with $r_{\mathrm{e}}$ in the wavelength regions (1) and (2), while for liquid clouds it varies the most with $r_{\mathrm{e}}$ in the wavelength region (3) (see Fig. 4). In the wavelength range (1), the local minimum observed for ice clouds deepens as cloud particle sizes increase, whereas liquid water cloud transmittance does not produce a local minimum. For the spectral range (2), radiance transmitted through a liquid cloud with distinct $r_{\mathrm{e}}$ (denoted by the thin lines with different shadings of blue in Fig. 4) varies only by the mean magnitude. In the same range, the peak signal at $1230 \mathrm{~nm}$ for ice clouds (denoted by different shadings of orange in Fig. 4) vanishes for largest $r_{\mathrm{e}}$, resulting in a concave shape. Radiance in (3) asymptotes to 0 for ice clouds but not for liquid water clouds. The magnitude and spectral slope of liquid water cloud normalized radiance in (3) are reduced as $r_{\mathrm{e}}$ increases.

\subsection{Definition of spectral parameters}

To quantify the spectral features discussed in Sect. 3.4, we introduce 15 parameters, designated by $\eta_{1}$ through $\eta_{15}$, with distinct spectral transmittance characteristics that will be used to infer cloud properties. The features represent the largest patterns of variations (outside of those due to water vapor) that were observed in a set of over 800000 cloud transmittance spectra spanning 15 days. In order to minimize the influence of water vapor on the cloud retrieval, wavelength ranges were restricted to those where water vapor absorption changed the signal by less than $5 \%$ for precipitable water ranging from 0 to $40 \mathrm{~mm}$. This is a far larger range of 


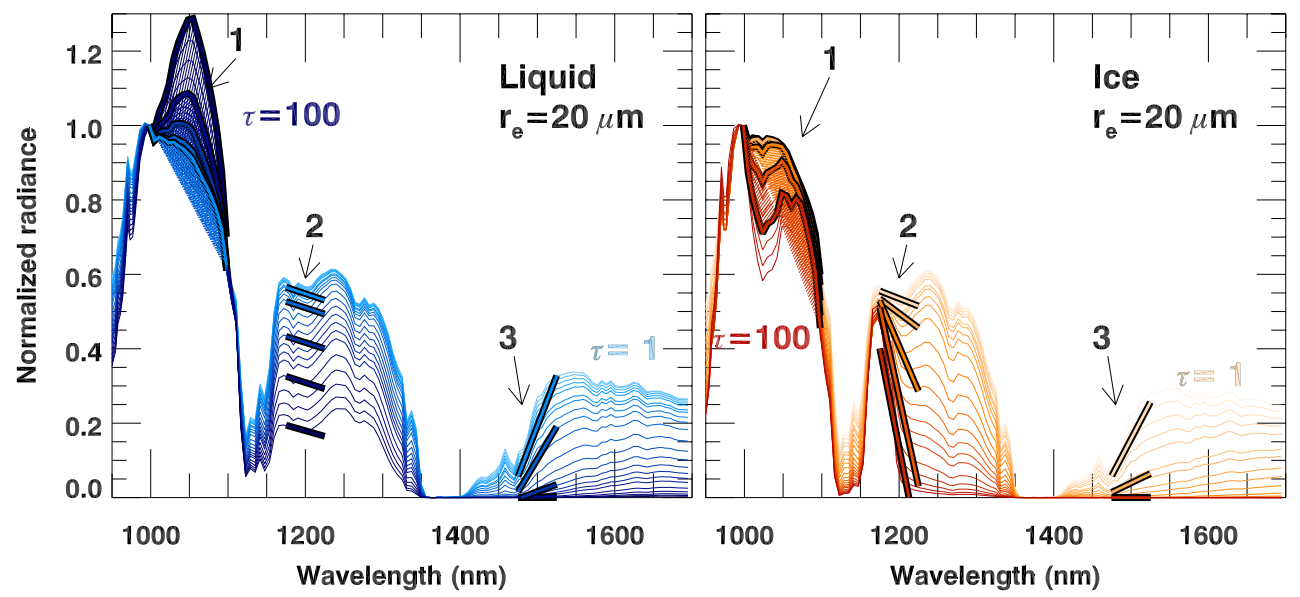

Figure 5. Radiance spectra normalized by its value at $1000 \mathrm{~nm}$ for liquid (left) and ice (right) clouds with $r_{\mathrm{e}}=20 \mu \mathrm{m}$ and $\tau$ varying from 1 to 100 (where darker colors denote optically thicker clouds). Three spectral features, which vary with changes of $\tau$ (see text), are quantified by the first three parameters, $\eta_{1}, \eta_{2}$, and $\eta_{3}$. The normalized radiance that contribute to calculating $\eta_{1}, \eta_{2}$, and $\eta_{3}$ are highlighted with a thicker line for a few radiance spectra with varying $\tau$ and are indicated by the numbers 1,2 , and 3 , respectively. The shaded areas indicated by 1 overlap for different optical thickness. To increase visibility of the smaller shaded areas (denoted by lighter blues and darker oranges), these are plotted in front of the larger shaded areas.

precipitable water observed over Boulder, where the mean is about $11 \mathrm{~mm}$.

The largest variability in spectral features is found in the three wavelength regions identified in Figs. 3 and 4 and has been quantified by 13 of the 15 parameters, where both absorption and scattering processes modulate transmittance. The transmitted radiance in a subset of wavelengths used to calculate the first three parameters are highlighted in Fig. 5. The first spectral feature is the peak radiance in the wavelength range (1). It is quantified by the curvature of the radiance spectrum, normalized by the radiance at $1000 \mathrm{~nm}$, and denoted by parameter 1 , or $\eta_{1}$. The curvature is calculated from the area bounded by the normalized radiance and a linear interpolation between 1000 and $1100 \mathrm{~nm}$ and is the sum of the difference between the normalized radiance and the linear interpolation at every wavelength. It is represented by the shaded area in Fig. 5. As $\tau$ increases, the shaded area for liquid clouds increases (for ice clouds it decreases), which consequently increases (decreases) $\eta_{1}$. The change in spectral normalized radiance transmitted through ice clouds in (2), which is transformed from convex to concave curvature around $1230 \mathrm{~nm}$ as $\tau$ increases, is quantified by the second parameter, $\eta_{2}$. This parameter is the spectral derivative of transmitted normalized radiance at $1200 \mathrm{~nm}$, at the edge of the convex/concave shape with a maximum/minimum at $1230 \mathrm{~nm} . \eta_{2}$ is identified by " 2 " and is highlighted to illustrate the trend with varying $\tau$ for ice clouds in Fig. 5. The spectral feature in (3), the curvature, is diminished as $\tau$ increases. This spectral feature is quantified with parameter $\eta_{3}$. $\eta_{3}$ is defined by the spectral derivative of transmitted normalized radiance at $1500 \mathrm{~nm}$, which decreases with increasing $\tau$ for both ice and liquid clouds.
Ten more parameters were defined to quantify the similar spectral features in the three wavelength ranges. $\eta_{7}, \eta_{9}, \eta_{12}$, and $\eta_{13}$ are defined within region (1) by the mean magnitude of normalized radiance $\left(\eta_{7}\right)$, the slope of the spectral derivative $\left(\eta_{9}\right)$, the value of the normalized radiance at one wavelength $\left(\eta_{12}\right)$, and the ratio of radiances at two different wavelengths $\left(\eta_{13}\right)$. In region (2), three additional parameters were defined by the ratio of radiances at two different wavelengths $\left(\eta_{4}\right)$, the mean magnitude of normalized radiance $\left(\eta_{5}\right)$, and the slope of the spectral derivative $\left(\eta_{10}\right)$. For region $(3), \eta_{8}$ quantifies the curvature by the same method described for $\eta_{1}$. In addition, $\eta_{6}$ and $\eta_{15}$ quantify the mean normalized radiance and the spectral slope in region (3), respectively. The equations used calculate all 15 spectral parameters, and short descriptions of the spectral parameter with expected behavior are listed in Table 1.

The characteristics in a fourth spectral region, between 530 and $610 \mathrm{~nm}$, exhibiting spectral variability in cloud transmittance are the basis for defining an additional parameter. For clouds with $\tau<4$, the spectral slope in this regions becomes progressively more negative and the magnitude of transmitted normalized radiance decreases as $\tau$ decreases, until it reduces to the clear sky spectrum (see Fig. 6a). $\eta_{11}$ quantifies the spectral slope of normalized radiance between $530 \mathrm{~nm}$ and $610 \mathrm{~nm}$, highlighted in Fig. 6a. The slopes calculated from these and other normalized spectra increase with $\tau$ until a maximum is reached (see Fig. 6b) at a value of $\tau \sim 3$ for ice clouds and $\tau$ up to 7 for liquid clouds. The spectral behavior in this fourth spectral region is modulated by changes in scattered radiation. 
Table 1. Definition, wavelength range used, and expected behavior for the 15 spectral parameters. $L_{\lambda}$ indicates the radiance evaluated at a wavelength, $\lambda$, in microns. The function $\operatorname{lin}(y ; \lambda=a . b)$ describes the linear fit of values evaluated at $\lambda=a$ and $\lambda=b$, where $y$ represents any variable that is calculated from the radiance spectrum. The function $\left.m(y)\right|_{\lambda=a} ^{b}$ describes the slope of $y$ evaluated over the range of $\lambda=a$ to $\lambda=b$. The average value of $y$ over the range of $\lambda=a$ to $\lambda=b$ is presented as $\left.\langle y\rangle\right|_{\lambda=a} ^{b}$. In addition to defining each spectral parameter by the symbol $\eta_{1}$ through $\eta_{15}$, other symbols are used to provide additional insight into the calculation of the parameter. For parameters defined by a measure of the curvature of radiance, $C_{\lambda}$ is used, where a convex curvature represents a slope that decreases with increasing wavelength and concave curvature for a slope that increases with increasing wavelength. The spectral derivatives of the normalized radiances are denoted by $\frac{\partial}{\partial \lambda_{\lambda}}$. When this derivative, as a function of wavelength, is fitted to a line, its slope is represented by $\frac{\partial^{2}}{\partial \lambda_{\lambda}^{2}}$. The slope of this derivative over a wavelength range is roughly equivalent to the average second derivative over the same wavelength range, but differences may result from the linear fitting process. Slopes, ratios, mean, and normalized radiances are presented as $\mathrm{sl}_{\lambda}, r_{\lambda},\langle R\rangle_{\lambda}$, and $R_{\lambda}$, respectively, with $\lambda$ denoting a representative wavelength in microns where the features are quantified.

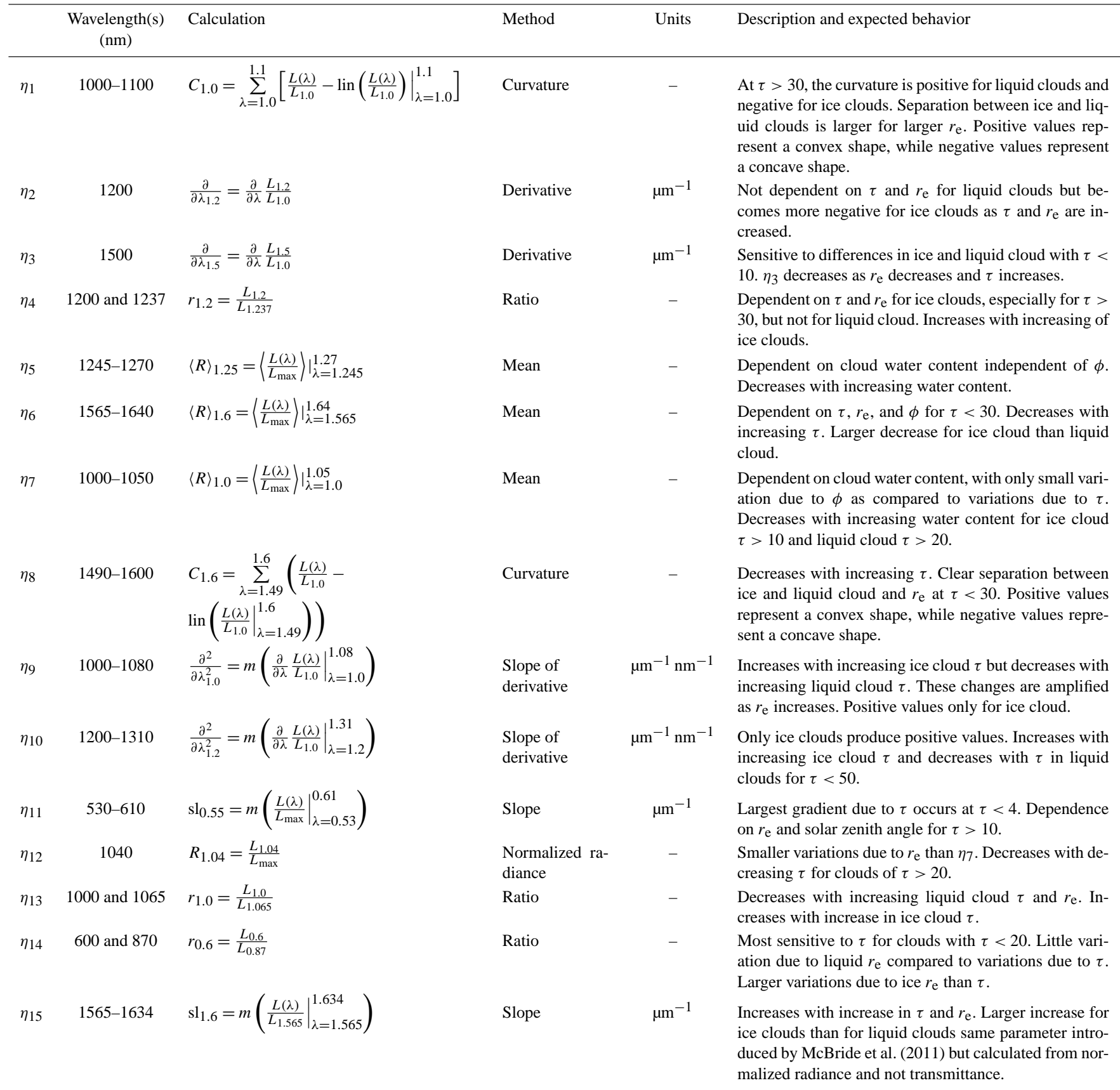


a)

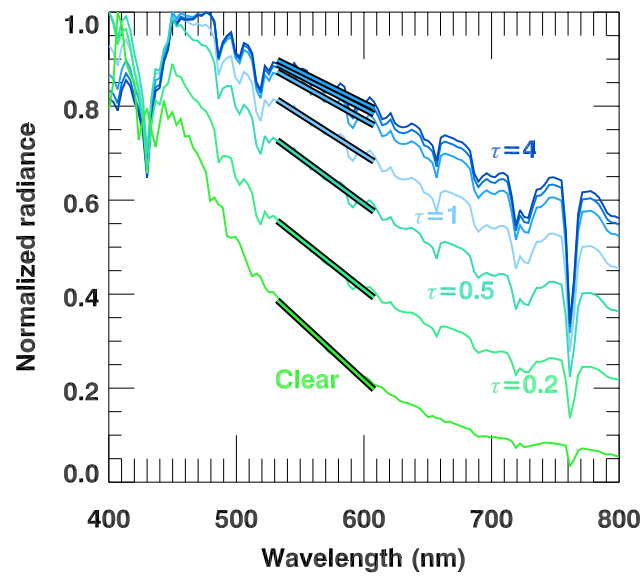

b)

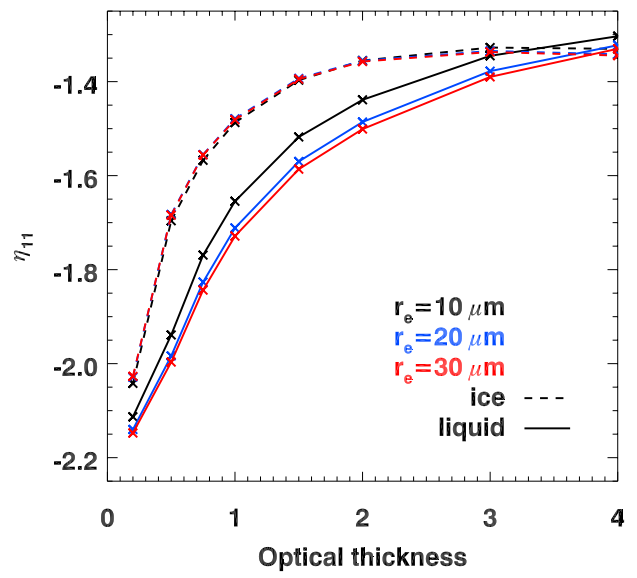

Figure 6. (a) Normalized transmitted radiance of liquid water clouds of varying optical thickness and effective radius of $20 \mu \mathrm{m}$ and (b) the slope of normalized radiance for the wavelength range of 530-610 nm, $\eta_{11}$, as a function of $\tau$ for ice and liquid clouds, evaluated for three different effective radii. In (a), the slope in the visible is identified by the highlighted region. The normalized radiance spectra and the $\eta_{11}$ calculated from them were modeled with ancillary inputs based on 25 May 2012 for a solar zenith angle of $50^{\circ}$.

\subsection{Dependence on thermodynamic phase, optical thickness, and effective radius}

The dependencies of the 15 parameters (Sect. 3.5; Table 1) on $\tau$ for ice clouds (dashed) and liquid clouds (solid) and for three different particle sizes are shown in Fig. 7. The accuracy for each of the parameters is estimated by propagating the measurement accuracy through the formula in Table 1. Modeled spectra are evaluated with the variation of SSFR's three radiometric calibrations and their related precision (Sect. 2.1). The standard deviation of the set of spectral parameters calculated from these modeled spectra is represented by the shaded area for each parameter in Fig. 7.

Parameter sensitivity to $\tau, r_{\mathrm{e}}$, and $\phi$ is larger than the measurement uncertainty (see Fig. 7) for several parameters, clearly establishing their suitability for distinguishing cloud properties. For clouds with $\tau<10$, the most appropriate parameters are $\eta_{3}, \eta_{8}$, and $\eta_{10}\left(\frac{\partial}{\partial \lambda_{1.5}}, C_{1.6}\right.$, and $\left.\frac{\partial^{2}}{\partial \lambda_{1.2}^{2}}\right)$. For clouds with $\tau>10$, the spectral parameters $\eta_{6}$ and $\eta_{15}$ $\left(\langle R\rangle_{1.6}\right.$ and $\left.\mathrm{sl}_{1.6}\right)$ have the largest sensitivity to $\tau$ and $r_{\mathrm{e}}$. The most suitable parameters for ice clouds are $\eta_{1}, \eta_{2}$, and $\eta_{9}$ $\left(C_{1.0}, \frac{\partial}{\partial \lambda_{1.2}}\right.$, and $\left.\frac{\partial^{2}}{\partial \lambda_{1.0}^{2}}\right)$. The largest sensitivity to $\tau$, regardless of $r_{\mathrm{e}}$ and $\phi$ is found in $\eta_{11}\left(\mathrm{sl}_{0.55}\right)$.

\subsubsection{Thermodynamic phase}

Thermodynamic phase is the largest contributor to the variability in $\eta_{1}, \eta_{2}, \eta_{4}, \eta_{9}, \eta_{10}$, and $\eta_{13}\left(C_{1.0}, \frac{\partial}{\partial \lambda_{1.2}}, r_{1.2}, \frac{\partial^{2}}{\partial \lambda_{1.0}^{2}}\right.$, $\frac{\partial^{2}}{\partial \lambda_{1.2}^{2}}$, and $r_{1.0}$, respectively). This is shown by the separability between the values of these parameters evaluated for a liquid cloud compared to an ice cloud (see Fig. 7). These parameters exploit spectral features that depend on cloud phase in the wavelength ranges (1) and (2); see Fig. 4. The differences between spectral features for each cloud thermodynamic phase are due to variations in absorption, which are more pronounced for the largest optical thicknesses, and changes in scattering properties to which clouds with the smallest optical thickness are the most sensitive.

The parameters $\eta_{1}, \eta_{9}$, and $\eta_{13}\left(C_{1.0}, \frac{\partial^{2}}{\partial \lambda_{1.0}^{2}}\right.$, and $\left.r_{1.0}\right)$ quantify spectral features in the wavelength range (1). Along with $\eta_{1}, \eta_{9}$ also calculates the curvature but by the slope of spectral derivative, where negative values of $\eta_{1}$ and positive values of $\eta_{9}$ are attained only by ice clouds. Values of $\eta_{13}$ lower than 1 are obtained by normalized radiance spectra transmitted through liquid clouds.

In the wavelength range (2), the transmittance for either ice and liquid water cloud result in large differences in the parameters $\eta_{2}, \eta_{4}$, and $\eta_{10}\left(\frac{\partial}{\partial \lambda_{1.2}}, r_{1.2}\right.$, and $\left.\frac{\partial^{2}}{\partial \lambda_{1.2}^{2}}\right)$. Values of $\eta_{2}$ for liquid water clouds are never lower than $-0.35 \mu \mathrm{m}^{-1}$ and vary little, whereas only ice clouds values as low as $-21.2 \mu \mathrm{m}^{-1}$ (Fig. 5). The large change in $\eta_{2}$ for ice clouds compared to liquid water clouds is due to a local maximum of absorption slightly longward of $1200 \mathrm{~nm}$ for ice but not for liquid water. These values hold for the ranges in surface albedo, cloud base height, atmospheric state, and solar zenith angle represented during the cases of 25 May 2012 and $6 \mathrm{Au}-$ gust 2012. Another measure of the spectral feature in (2) is the ratio of radiance at 1200 and $1237 \mathrm{~nm}\left(\eta_{4}\right)$, which increases to as high as 18.8 with increases in ice cloud $\tau$ but varies much less with changes in liquid water cloud properties. The signal-to-noise ratio of $\eta_{4}$ is reduced below 1 for large ice cloud $\tau$ where the radiance at $1237 \mathrm{~nm}$ is entirely attenuated. The curvature of normalized radiance in (2), quantified by $\eta_{2}$, is also quantified by the slope of the 

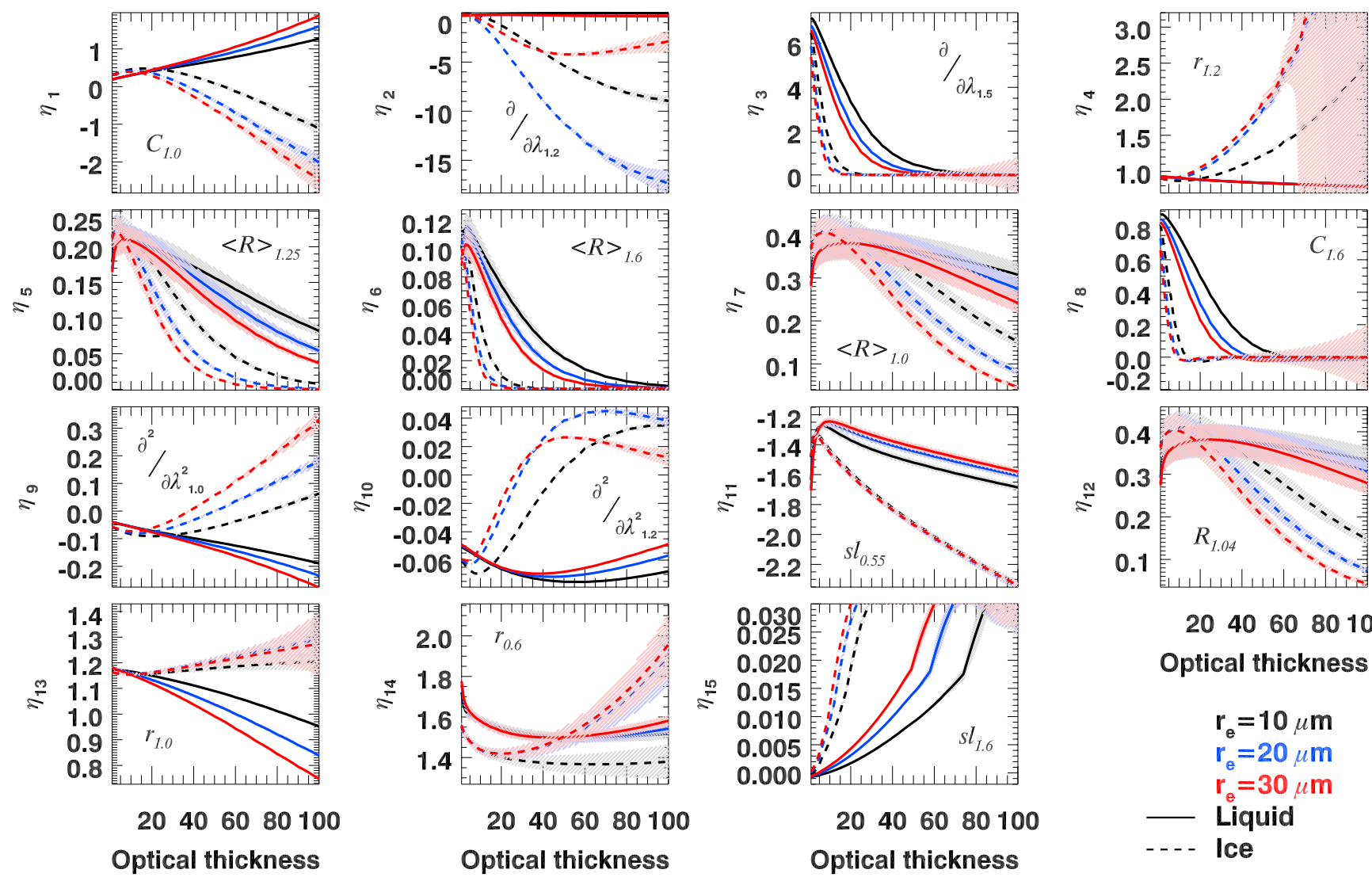

20406080100 Optical thickness

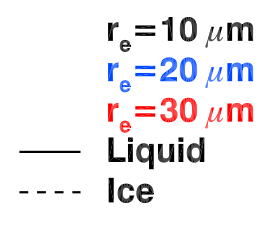

Figure 7. The value of the parameters $\eta_{1}$ through $\eta_{15}$ as a function of optical thickness for three effective radii (colors) for liquid (solid) and ice (dashed) clouds. The shading surrounding each line denotes the standard deviation of the variability in the parameters due to measurement uncertainty (see text).

spectral derivative, $\eta_{10}$, which increases (decreases) with optical thickness for ice (liquid) clouds. When transmittance is nearly entirely attenuated ( $\tau>40)$, the concave and convex shapes become less pronounced; therefore, $\eta_{10}$ decreases for ice clouds and increases for liquid clouds.

\subsubsection{Optical thickness}

Parameters $\eta_{3}, \eta_{5}, \eta_{6}, \eta_{7}, \eta_{8}, \eta_{11}, \eta_{12}, \eta_{14}$, and $\eta_{15}\left(\frac{\partial}{\partial \lambda_{1.5}}\right.$, $\langle R\rangle_{1.25},\langle R\rangle_{1.6},\langle R\rangle_{1.0}, C_{1.6}, \mathrm{sl}_{0.55}, R_{1.04}, r_{0.6}$, and $\left.\mathrm{sl}_{1.6}\right)$ are most sensitive to changes in $\tau$. At $\tau<5$, scattered radiation is the primary contributor to change in $\eta_{5}, \eta_{6}, \eta_{7}, \eta_{11}, \eta_{12}$, and $\eta_{14}$, and it varies with $\tau$ (see Fig. 7). Of these parameters, $\eta_{5}, \eta_{6}, \eta_{7}$, and $\eta_{12}$ quantify the magnitude of transmitted normalized radiance in all three wavelength ranges. Of the remaining parameters $\eta_{14}$, quantifies the same spectral feature presented in Fig. 6 and is quantified by $\eta_{11}$. Unlike $\eta_{11}$, the influence of the underlying surface albedo on $\eta_{14}$ increases with increasing $\tau$ at $\tau>5$, as expected by the physical process described by Chiu et al. (2006).

For $\tau>5$, the spectral features that are quantified by $\eta_{3}$, $\eta_{5}, \eta_{6}, \eta_{7}, \eta_{8}$, and $\eta_{12}\left(\frac{\partial}{\partial \lambda_{1.5}},\langle R\rangle_{1.25},\langle R\rangle_{1.6},\langle R\rangle_{1.0}, C_{1.6}\right.$, and $\left.R_{1.04}\right)$ are increasingly dominated by absorption. This causes a reduction in the magnitude of normalized radiance, evaluated by $\eta_{5}, \eta_{6}, \eta_{7}$, and $\eta_{12} . \eta_{3}, \eta_{8}$, and $\eta_{15}\left(\frac{\partial}{\partial \lambda_{1.5}}, C_{1.6}\right.$, and $\mathrm{sl}_{1.6}$ ) quantify the change in (3) due to ice and liquid water absorption, which is enhanced with increasing $\tau$ due to higher-order scattering. When the shape of transmitted normalized radiance flattens, $\eta_{3}$ and $\eta_{8}$ decrease while $\eta_{15}$ increases.

\subsubsection{Effective radius}

Effective radius contributes to variability in all 15 parameters. In particular, $\eta_{1}$ to $\eta_{10}, \eta_{12}, \eta_{13}$, and $\eta_{15}$ quantifies the spectral features that are linked to absorption modified by $r_{\mathrm{e}}$, while $\eta_{3}$ and $\eta_{5}$ to $\eta_{8}, \eta_{11}$, and $\eta_{15}$ quantifies the changes in the spectral features due to the changes in scattering properties of different $r_{\mathrm{e}} . \eta_{1}, \eta_{2}, \eta_{3}, \eta_{4}, \eta_{9}, \eta_{10}, \eta_{13}$, and $\eta_{15}\left(C_{1.0}\right.$, $\frac{\partial}{\partial \lambda_{1.2}}, \frac{\partial}{\partial \lambda_{1.5}}, r_{1.2}, \frac{\partial^{2}}{\partial \lambda_{1.0}^{2}}, \frac{\partial^{2}}{\partial \lambda_{1.2}^{2}}, r_{1.0}$, and $\left.\mathrm{sl}_{1.6}\right)$ quantify spectral features that are unique to absorption, which increases with particle size at any one $\tau$. In the wavelength range (1), the separation between liquid water and ice values of $\eta_{1}, \eta_{9}$, and $\eta_{13}$ increases with $r_{\mathrm{e}} . \eta_{2}, \eta_{4}$, and $\eta_{10}$ parameterize the transmittance feature in (2) linked to $r_{\mathrm{e}}$-dependent ice particle 
absorption but not liquid water absorption. For $\eta_{15}$, absorption decreases from $1565 \mathrm{~nm}$ towards longer wavelengths (McBride et al., 2011), resulting in a dependence on $r_{\mathrm{e}}$, also quantified by $\eta_{3}$ in the same wavelength region. Cloud particle size affects $\eta_{3}, \eta_{5}, \eta_{6}, \eta_{7}, \eta_{8}$, and $\eta_{12}\left(\frac{\partial}{\partial \lambda_{1.5}},\langle R\rangle_{1.25}\right.$, $\langle R\rangle_{1.6},\langle R\rangle_{1.0}, C_{1.6}$, and $\left.R_{1.04}\right)$ by modulating the spectral features through scattering and absorption. These spectral parameters are consistently higher at any one $\tau$ and $\phi$ for the smallest $r_{\mathrm{e}}$, regardless if it is scattering or absorption that dominates.

\section{Retrieval methodology}

For any zenith radiance spectrum, a single spectral parameter is insufficient to derive $\tau, r_{\mathrm{e}}$, and $\phi$. Multiple spectral parameters can be used to first identify thermodynamic phase $(\phi)$, followed by another procedure employing additional parameters to derive $\tau$ and $r_{\mathrm{e}}$. Eq. (1) is the statistic, $\chi^{2}\left(\tau, r_{\mathrm{e}}, \phi\right)$, used to retrieve $\tau$ and $r_{\mathrm{e}}$ from a weighted least-squares fit between modeled and observed values of all 15 parameters $(i=1,2,3, \ldots, 15)$. The retrieval is defined at the closest match (minimum in $\chi^{2}$ ) between measurement-derived parameters, $\eta_{i}$, and the parameters derived from forward-model LUT $\eta_{i}^{*}\left(\tau, r_{\mathrm{e}}, \phi\right)$ at cosine of the solar zenith angle closest to that of the observation.

$\chi^{2}\left(\tau, r_{\mathrm{e}}, \phi\right)=\sum_{i=1}^{15} \frac{1}{w_{i}}\left(\boldsymbol{\eta}_{i}-\boldsymbol{\eta}_{i}^{*}\left(\tau, r_{\mathrm{e}}, \phi\right)\right)^{2}$

In order to properly weight the contributions from each parameter, a factor, $w_{i}$, is derived from the full range of the parameters, $P_{i}$. Not all parameters can be determined with equal certainty; therefore, the measurement uncertainty, $\Delta \eta_{i}$, of each parameter is also included in the weighting factor in a manner similar to Wan and Li (1997), where the most uncertain parameter has lowest influence on the solution.

$w_{i}=\Delta \boldsymbol{\eta}_{i} P_{i}$

$P_{i}=\max \left(\eta_{i}^{*}\left(\tau, r_{\mathrm{e}}, \phi\right)\right)-\min \left(\eta_{i}^{*}\left(\tau, r_{\mathrm{e}}, \phi\right)\right)$

Therefore, the maximum contribution of each parameter to $\chi^{2}\left(\tau, r_{\mathrm{e}}, \phi\right)$ is 1 ; the theoretical maximum value of $\chi^{2}\left(\tau, r_{\mathrm{e}}, \phi\right)$ is 15 . The full range of each parameter is obtained by the maximum and minimum of the LUTs.

The retrieval process consists of two steps. In the first step, thermodynamic phase, $\phi$, can be discriminated by using a single parameter or with the combination of all 15 parameters. $\eta_{1}, \eta_{2}, \eta_{4}, \eta_{9}, \eta_{10}$, and $\eta_{13}\left(C_{1.0}, \frac{\partial}{\partial \lambda_{1.2}}, r_{1.2}, \frac{\partial^{2}}{\partial \lambda_{1.0}^{2}}, \frac{\partial^{2}}{\partial \lambda_{1.2}^{2}}\right.$, and $\left.r_{1.0}\right)$ return unique values for either ice or liquid clouds for clouds with optical thickness larger than 10. Positive values for $\eta_{9}$ and $\eta_{10}$, negative values for $\eta_{1}$, and values below $-0.35 \mu \mathrm{m}^{-1}$ for $\eta_{2}$ are unique to ice clouds for the environmental conditions (ancillary inputs) described in Sect. 3.2. If any of these six parameters have values unique to ice clouds with $\tau>10$, the cloud thermodynamic phase is therefore ice, and the retrieval process moves on to the second step. Outside this range, both ice and liquid water clouds produce the same parameters, and thus $\phi$ cannot be discriminated with a single parameter. For all clouds with $\tau$ lower than 10 (as $r_{\mathrm{e}}$ increases, this cutoff in $\tau$ decreases), a combination of all 15 parameters is used to determine $\phi$ by the best-fit LUT (described in Sect. 3.3) solution identified at the minimum $\chi^{2}$ defined in Eq. (1). Although multiple parameters are required to discriminate $\phi$, this does not mean that $\phi$ is any less certain than when using a single spectral parameter. The thermodynamic phase retrieval is bimodal and determines if the cloud is either pure ice or pure liquid, not a ratio of the two.

Once $\phi$ is determined, a second step consists of a $\phi$ segregated retrieval of $\tau$ and $r_{\mathrm{e}}$. To apply the $\phi$-segregated retrieval, we use the LUT of the appropriate $\phi$ : either $\boldsymbol{\eta}_{i}^{*}\left(\tau, r_{\mathrm{e}}, \phi=\right.$ ice $)$ or $\boldsymbol{\eta}_{i}^{*}\left(\tau, r_{\mathrm{e}}, \phi=\right.$ liquid $)$. The LUTs for ice or liquid clouds are defined with different ranges in $r_{\mathrm{e}}$, which represent naturally occurring ice or liquid cloud particle size (see Sect. 3.3). The retrieved $\tau$ and $r_{\mathrm{e}}$ represents the location in the LUT which defines minimum $\chi^{2}$, calculated from Eq. (1).

When determining $\chi^{2}$, some of the 15 parameters calculated from the measured spectrum do not fall within the range of the LUT. The parameters that do not fall within the LUT are deemed non-physical and are therefore omitted from the calculation of $\chi^{2}$. Values outside the LUT occur when the signal-to-noise ratio of the parameter is lower than one, or it is at the limit of ranges within the LUT. An example of small signal-to-noise ratio occurs for $\eta_{4}$ when evaluated for ice clouds with $\tau>65$ and $r_{\mathrm{e}}>30 \mu \mathrm{m}$; see the large shaded area in Fig. 7. Since each parameter results from different physical processes, the signal-to-noise ratio may be lower than one for certain parameters but not for others (for example, $\eta_{4}$ compared to $\eta_{9}$ for ice clouds with $\tau>65$ ). Other examples of more noise than signal can be identified when the shaded areas in Fig. 7 extends past the modeled value. This occurs for $\eta_{8}$ when it is lower than -0.1 or for $\eta_{3}$ when it is lower than -0.5 . Therefore, the solution may still be valid even without the contributions from uncertain parameters.

A successful retrieval occurs when the parameters calculated from a measurement spectrum matches those modeled for at least one combination of $\tau, r_{\mathrm{e}}$, and $\phi$. This match is defined where the minimum $\chi^{2}$ is lower than predefined value, 0.69 . This value represents $4.6 \%$ of the total theoretical maximum $\chi^{2}$, which is the same percentage as the measurement uncertainty in normalized radiance spectra (see Sect. 2.1).

\subsection{Retrieval uncertainty}

To obtain the retrieval uncertainty of $\tau$ and $r_{\mathrm{e}}$, we propagate measurement uncertainty through the retrieval equation. While uncertainties in ancillary inputs or multilayered clouds may occur, this section only refers to uncertainties related to 
known variations in measurements linked to calibration stability and noise variance. These uncertainties are not representative of possible systematic problems linked to calibration biases, such as differences between measured radiances from the two different spectrometers used in the SSFR. The uncertainty in the parameters, $\Delta \eta_{i}$, and the slope of $\chi^{2}$ as a function of the parameters quantify the expected variation in $\chi^{2}$ resulting from measurement uncertainty, described by $\Delta \chi^{2}$ (adapted from Taylor, 1997).

$\Delta \chi^{2}\left(\tau, r_{\mathrm{e}}, \phi\right)=\sqrt{\sum_{i=1}^{15}\left(\frac{\partial \chi^{2}\left(\tau, r_{\mathrm{e}}, \phi\right)}{\partial \eta_{i}} \Delta \eta_{i}\right)^{2}}$

This expected variation in $\chi^{2}$ due to measurement uncertainty $\left( \pm \Delta \chi^{2}\right)$ results in a range of $\tau$ and $r_{\mathrm{e}}$ where $\chi^{2} \pm \Delta \chi^{2}$ is minimized. The minimum $\chi^{2}-\Delta \chi^{2}$ (or $\chi^{2}+\Delta \chi^{2}$ ) occurs at the combination of $\left.\tau\right|_{\chi^{2}-\Delta \chi^{2}}\left(\right.$ or $\left.\left.\tau\right|_{\chi^{2}+\Delta \chi^{2}}\right)$ and $\left.r_{\mathrm{e}}\right|_{\chi^{2}-\Delta \chi^{2}}$ (or $\left.r_{\mathrm{e}}\right|_{\chi^{2}+\Delta \chi^{2}}$ ). The difference between $\tau$ and $r_{\mathrm{e}}$ evaluated at the extremes of the range of variability of $\chi^{2}$ represent their respective uncertainty, $\Delta \tau$ and $\Delta r_{\mathrm{e}}$.

$$
\begin{aligned}
& \Delta \tau=\frac{1}{2}|\tau|_{\chi^{2}-\Delta \chi^{2}}-\left.\tau\right|_{\chi^{2}+\Delta \chi^{2}} \mid \\
& \Delta r_{\mathrm{e}}=\frac{1}{2}\left|r_{\mathrm{e}}\right|_{\chi^{2}-\Delta \chi^{2}}-\left.r_{\mathrm{e}}\right|_{\chi^{2}+\Delta \chi^{2}} \mid
\end{aligned}
$$

\subsection{Comparisons to other methods}

The retrieval described herein is compared to two other methods: the method developed by McBride et al. (2011), hereafter named the slope method, and the standard twowavelength method often used in reflectance (Nakajima and King, 1990) but applied to transmittance as described by Kikuchi et al. (2006), hereafter named the two-wavelength method.

\subsubsection{Slope method}

The slope method uses transmittance at $515 \mathrm{~nm}$ and the slope of transmittance at $1565-1634 \mathrm{~nm}$ normalized by the transmittance value at $1565 \mathrm{~nm}$ to retrieve $\tau$ and $r_{\mathrm{e}}$. A leastsquares fit matches measured transmittance and slope values to a LUT containing modeled transmittances and slopes for a set of $\tau$ and $r_{\mathrm{e}}$. The retrieval is successful if the uncertainty in $r_{\mathrm{e}}$ is smaller than $2 \mu \mathrm{m}$. The LUTs used for this retrieval method are based on the same radiative transfer calculations introduced in Sect. 3.1. For case A, the slope method is applied using a liquid cloud LUT, while for case $\mathrm{C}$ an ice cloud LUT is used. For case B, the retrieved thermodynamic phase using the method described in Sect. 4 dictates which LUT is used: either ice or liquid cloud LUT. Case C represents the first time the method described by McBride et al. (2011), is applied to an ice cloud. For this case, all retrieved values are presented, even if the uncertainty in $r_{\mathrm{e}}$ is larger than $2 \mu \mathrm{m}$.

\subsubsection{Two-wavelength method}

The two-wavelength method retrieves $\tau$ and $r_{\mathrm{e}}$ using transmittance at wavelengths in the mid-visible $(515 \mathrm{~nm})$ and in the NIR $(1630 \mathrm{~nm})$ with an LUT, similarly to the current work's method and the slope method. Kikuchi et al. (2006) employed transmittance evaluated at 1020 and $1600 \mathrm{~nm}$. For this work, we follow the description of the "standard" method presented by McBride et al. (2011), which employs transmittance at 515 and $1630 \mathrm{~nm}$ to retrieve $\tau$ and $r_{\mathrm{e}}$. Although the two-wavelength method produces large uncertainties in $r_{\mathrm{e}}$ when applied to clouds with $\tau<25$, we still apply it here and report the associated uncertainties, which can be as large as $\pm 6.5 \mu \mathrm{m}$. The uncertainties in $\tau$ and $r_{\mathrm{e}}$ are calculated by the same method described by McBride et al. (2011) but applied to the measurement uncertainty of the SSFR $(8 \%)$ used in this work. Similarly to the slope method, the twowavelength retrieval employs a liquid water cloud LUT for case A, an ice cloud LUT for case C, and an ice or a liquid cloud for case B depending on the retrieved thermodynamic phase dictated by the current work's retrieval.

\subsubsection{Comparison of time series}

Figure 8 shows the time series of $\tau, r_{\mathrm{e}}$, and $\phi$ retrieved using the 15-parameter method (this work), the slope retrieval, and the two-wavelength retrieval based on transmitted radiance for the (A) liquid, (B) mixed-phase, and (C) ice cloud cases.

The ancillary data (discussed in Sect. 3.2) and the 15parameter method classified each case consistently with respect to the thermodynamic phase. The $\tau$ retrieved with all three methods follows similar trends albeit with different magnitudes. The retrieved $r_{\mathrm{e}}$ based on all three methods also follow the similar trends; although the two-wavelength method in case $\mathrm{C}$ is anomalous. In addition, the uncertainty in the 15-parameter method retrievals is lower than that for the other two methods, especially for case $\mathrm{C}$, with an average $r_{\mathrm{e}}$ uncertainty of $\pm 1.2 \mu \mathrm{m}$, smaller by a factor of at least 2.5 for the slope and two-wavelength method.

For case $\mathrm{A}$, the 15-parameter method retrieved $\tau$ matches more closely to the two-wavelength method (mean difference of 2.5) than the slope method (mean difference of 5.6), while the retrieved $r_{\mathrm{e}}$ matches more closely the slope method (mean difference of $2.6 \mu \mathrm{m}$ ) then the two-wavelength method (mean difference of $4.4 \mu \mathrm{m}$ ). The largest differences occur between $\tau$ retrieved with the 15-parameter method and $\tau$ retrieved with the slope method whenever the 15-parameter $\tau$ is larger than 30. The range of $\tau$ retrieved with all three methods is never larger than 8 for the times between 15.0 and 15.4 UTC. This period coincides with times where the uncertainty in $\tau$ for the 15-parameter method is lowest, with a mean of \pm 1.2 and the average retrieved $\tau$ is highest at 32. This mean uncertainty increases to \pm 2.6 for later times, where the average $\tau$ decreases to 28 . The slope and twowavelength method retrieved an uncertainty of $\tau$ consistent 

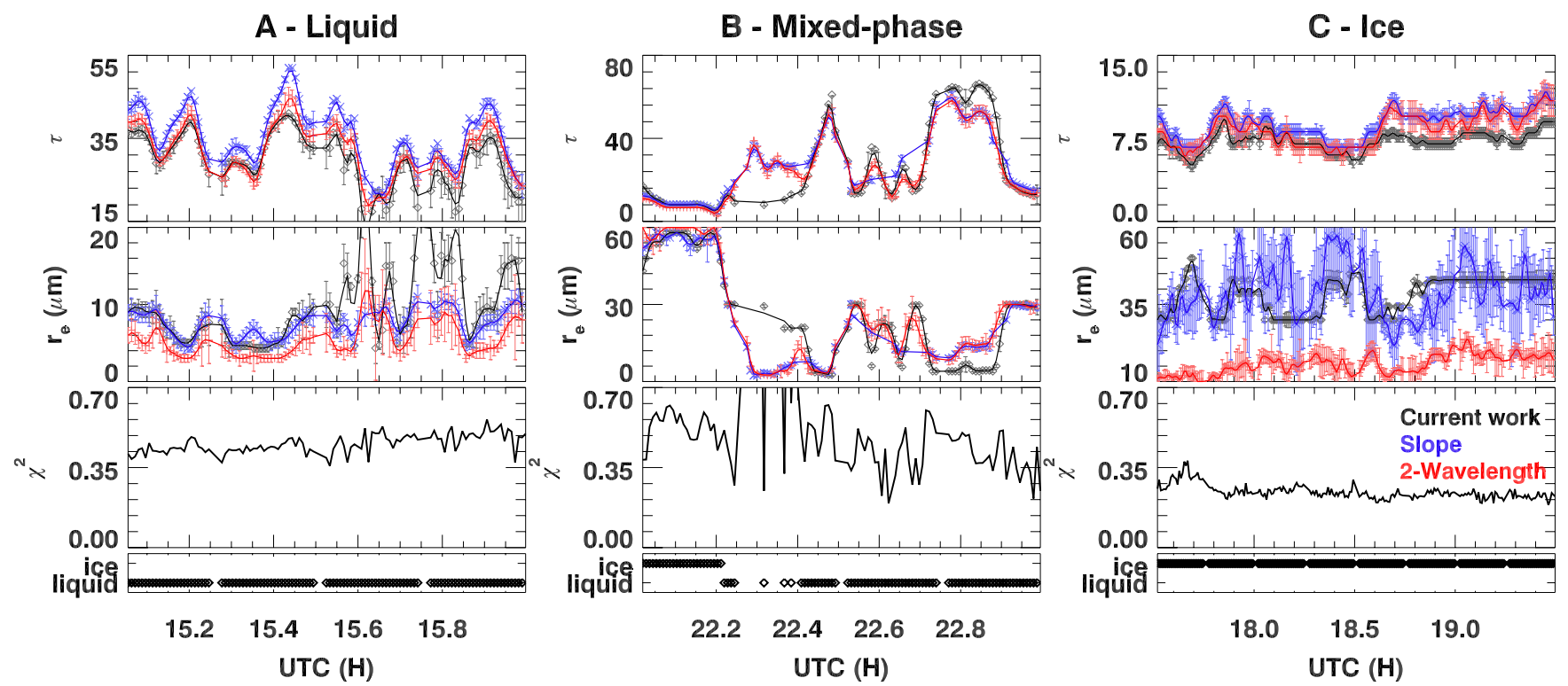

Figure 8. Time series of retrieved cloud properties determined by three different cloud-transmitted radiance-based retrieval methods: case A (left) for liquid cloud, case B (center) for mixed-phase cloud, and case C (right) for ice cloud. The mixed-phase cloud case represents a cloud sampled during a time where both ice and liquid water cloud particles are expected to have been present. Colors represent three different cloud retrieval methods: this work (black), the slope method (blue), and the two-wavelength method (red). Each point and uncertainty range represent one single retrieval, while the lines represent a three-point smoothed curve for illustrating the trend. For each case study, the top-most panel presents the retrieved optical thickness, $\tau$; the panel directly below shows the effective radius, $r_{\mathrm{e}}$; the panel directly below shows the minimum $\chi^{2}$ determined by this work (15-parameter method); and the lowest panel shows thermodynamic phase, $\phi$, designated by this work. The $y$ axis scale in $\tau$ and $r_{\mathrm{e}}$ differs for each case.

throughout the measurement period, with averages of \pm 1.3 and \pm 1.5 , respectively. Additional comparisons for this case are presented below.

Similarly to the comparison of $\tau$ for case A, the effective radii retrieved by all three methods matches more closely (within $5 \mu \mathrm{m}$ ) during the period from 15.0 to 15.4 UTC than the later period (within $10 \mu \mathrm{m}$ ). The uncertainty of $r_{\mathrm{e}}$ for all three methods is also lower in the earlier period (a mean of $\pm 1 \mu \mathrm{m}$ for all three methods) than the later period (a mean of $\pm 1.9 \mu \mathrm{m})$. Higher-than-average $r_{\mathrm{e}}$ uncertainty coincides with retrieved- $\tau$ lower than 20 for all three methods. Such behavior for low $\tau$ has been observed previously for the slope and two-wavelength method (Coddington et al., 2013; McBride et al., 2011). The $r_{\mathrm{e}}$ retrieved with the current work is within the uncertainty range of $r_{\mathrm{e}}$ retrieved with the two-wavelength method for $27 \%$ and the slope method for $69 \%$ of the time series.

Case B presents examples of retrieval behavior under conditions of liquid and ice cloud particle absorption. Although we have not addressed the applicability of the 15 spectral parameters to mixed phase clouds in this work, we investigate the results of the thermodynamic phase discrimination and the residual of least-squares fit of the retrieval to measured zenith radiances under conditions of concurrent ice and liquid absorption. This mixed-phase case is also used to present the extent of disagreement between retrievals when the cloud's thermodynamic phase is neither pure ice nor pure liquid water. The thermodynamic phase retrieval coincides with the thermodynamic phase retrieved by GOES, which was ice prior to, and liquid during, the measurement period. The largest $\chi^{2}$ occurs between 22.2 UTC and 22.4 UTC, when the thermodynamic phase transitions from an ice cloud to a liquid cloud, as determined by the 15-parameter method. In this transition zone, only $40 \%$ of the solutions obtained by the retrieval described herein are valid. Large residuals indicate that more information is required to reproduce the parameters calculated from the measurements of radiance spectra. Beyond this transition period, the match between $\tau$ retrieved from all three methods is exceptional, especially since none of the retrievals explicitly takes into account mixedphase clouds. The overall trend is reproduced with all three retrieval methods, and they differ by an average of 5.8 in retrieved $\tau$ and $4.7 \mu \mathrm{m}$ in retrieved $r_{\mathrm{e}}$ for the period later than 22.4 UTC. At these times, uncertainties in $r_{\mathrm{e}}$ are greater than $\pm 2 \mu \mathrm{m}$ for $13 \%$ of the time series for the current method, $26 \%$ for the slope method, and $44 \%$ for the two-wavelength method.

For case $\mathrm{C}$, the match in $\tau$ retrieved with all three methods differs by less 3 throughout the entire time series even though the retrieved $r_{\mathrm{e}}$ differs by as much as $37 \mu \mathrm{m}$. The $r_{\mathrm{e}}$ uncertainty is larger than $\pm 2 \mu \mathrm{m}$ at all times for the slope method, $62 \%$ of the time series for the two-wavelength method, and 
$29 \%$ of the time series when retrieved with the 15-parameter method. These large uncertainties obtained from the slope and two-wavelength method are likely due to a lower signalto-noise ratio of ice cloud transmitted radiance compared to a liquid water cloud transmitted radiance near $1600 \mathrm{~nm}$. This low signal can be observed in ice cloud radiance spectrum for a $\tau=10$ with $r_{\mathrm{e}}=20 \mu \mathrm{m}$ (Fig. 2a). The smaller $r_{\mathrm{e}}$ uncertainties obtained by the 15-parameter method compared to the slope and two-wavelength retrieval is also expected. Specific parameters were designed to take advantage of features that have a high signal-to-noise ratio for ice and liquid clouds (e.g., $\eta_{1}\left(C_{1.0}\right), \eta_{2}\left(\frac{\partial}{\partial \lambda_{1.2}}\right), \eta_{9}\left(\frac{\partial^{2}}{\partial \lambda_{1.0}^{2}}\right), \eta_{10}\left(\frac{\partial^{2}}{\partial \lambda_{1.2}^{2}}\right)$, and $\left.\eta_{13}\left(r_{1.0}\right)\right)$.

During the ice cloud case C, MODIS observed the same cloud at 18.17 UTC. Keep in mind that $\tau$ and $r_{\mathrm{e}}$ retrieved by MODIS were from a sampling volume close to cloud top, unlike the transmitted-radiance-based 15-parameter method. Note also the spatial resolution of MODIS, $1 \mathrm{~km}^{2}$, vs. that of the zenith-pointing SSFR, estimated to be $0.45 \mathrm{~km}^{2}$ for a cloud base at $7.5 \mathrm{~km}$. At $18.17 \mathrm{UTC}$, the 15 -parameter method retrieved $\tau=8 \pm 0.5$ and $r_{\mathrm{e}}=30 \pm 2 \mu \mathrm{m}$, while MODIS retrieved $\tau=12.7 \pm 15.3$ and $r_{\mathrm{e}}=17.1 \pm 5.4 \mu \mathrm{m}$ with "very good confidence" for both $\tau$ and $r_{\mathrm{e}}$ retrievals (Platnick et al., 2003). The 15-parameter $\tau$ retrieval falls within the uncertainty range of MODIS. The difference in retrieved $r_{\mathrm{e}}$ is likely due to the difference between particle sizes at cloud top and particle sizes throughout the cloud. Both the slope and two-wavelength methods resulted in a retrieval with an $r_{\mathrm{e}}$-uncertainty larger than $2 \mu \mathrm{m}$ at this time. Another possible cause of differences between these methods may be due to differing effects of ice crystal shapes, which alters the scattering properties of ice clouds. For reflectancebased retrievals, a change in assumed ice crystal roughness, from moderate to severe for ice particles with $r_{\mathrm{e}}=20 \mu \mathrm{m}$, increases the calculated reflectance at $1640 \mathrm{~nm}$ by $10 \%$ (Baum et al., 2011). Such changes in ice crystal shape to transmitted radiance is subject to further research.

\subsubsection{Comparison of retrieved $\tau$ and $r_{\mathrm{e}}$ for the liquid cloud case}

All three retrievals for case A exhibit a high degree of correlation. The linear correlation coefficient $\left(R^{2}\right)$ between the 15-parameter method and the other methods is 0.95 for $\tau$ but lower for $r_{\mathrm{e}}$ (see Fig. 9). The $\tau$ retrieved with the slope and two-wavelength methods is consistently higher than the 15 -parameter $\tau$. The difference between the 15-parameter method and the two other methods increases linearly with increasing $\tau$ by a ratio of 1.28 and 1.10 for the slope and two-wavelength methods, respectively (Fig. 9a). In addition, the intercept of the slope indicates that the 15-parameter has a bias of +1 and +3 with respect to the two-wavelength and slope method, respectively. For $r_{\mathrm{e}}$ (Fig. 9b), the twowavelength method retrieves lower $r_{\mathrm{e}}$ than the 15-parameter
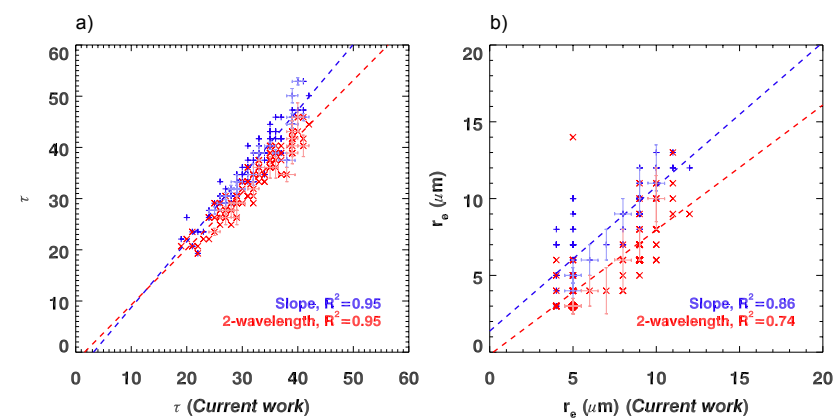

Figure 9. Scatter plot of (a) optical thickness and (b) effective radius for the liquid water cloud (case A) from the slope (blue) and two-wavelength (red) methods compared to the current work's retrieval. Dashed lines represent the best-fit lines. Larger thickness of the symbols indicates higher density of the points at that location. Uncertainty bars are shown on only a few points as to not overwhelm the figure.

method by a ratio of 0.81 ; the slope method does not have such a low bias with respect to $r_{\mathrm{e}}$ retrieved by the current method, which is supported by the intercept of the fitted line. A greater correlation between $\tau$ than $r_{\mathrm{e}}$, retrieved with the different methods, was also observed by McBride et al. (2011) for the two-wavelength and slope retrievals.

For this liquid cloud case, factors contributing to differences in retrieved $r_{\mathrm{e}}$ values include varying effects of vertical profiles in $r_{\mathrm{e}}$ evaluated at different wavelengths (Platnick, 2000), horizontal cloud inhomogeneities (e.g., Iwabuchi and Hayasaka, 2002; Marshak et al., 2006), and undetected presence of ice crystals (Sun and Shine, 1994). Although transmitted radiation interacts with cloud particles throughout the vertical extent of a cloud, this interaction is not the same for radiance at all wavelengths. Platnick (2000) shows that cloud particles in the lowest part of the cloud have a greater influence on transmittance for wavelength at $3700 \mathrm{~nm}$ than at $2200 \mathrm{~nm}$ and even less influence on transmittance at $1600 \mathrm{~nm}$. Those cloud particles are responsible for changes in transmitted irradiance of up to $6 \%$ at those wavelengths. This would suggest that cloud retrievals based on transmittance at shorter wavelengths would be less dependent on absorption and scattering by cloud particles in the lowest part of the cloud than transmittance at longer wavelengths. Although the influence of the cloud particles near cloud base on the different parameters has not been determined, one would expect that the parameters based on mean normalized radiance at wavelengths shorter than $1600 \mathrm{~nm}$ would be less influenced by particles at cloud base than the two-wavelength method. For liquid clouds, the smallest cloud particles are often located near base (e.g., Zhang et al., 2011); therefore, the 15-parameter method would retrieve larger $r_{\mathrm{e}}$ than the two-wavelength method. For case A, the 15-parameter method retrieved larger $r_{\mathrm{e}}$ than the twowavelength method for $81 \%$ of the times, which could in 
Table 2. Root mean square of the percent difference for the entire wavelength range between modeled spectra based on the three different retrievals and the three sample measured spectra presented in Fig. 10.

\begin{tabular}{lccc}
\hline & Current work $(\%)$ & Slope $(\%)$ & two-wavelength $(\%)$ \\
\hline Case A & 3.1 & 6.4 & 5.9 \\
Case B & $7.2(22.8$ for ice $)$ & 13.3 & 7.3 \\
Case C & 5.9 & 22.5 & 20.2 \\
\hline
\end{tabular}

part be due to the spectral dependence of vertical distribution of cloud particle size on transmitted radiance. In addition, Kikuchi et al. (2006), showed that clouds with vertically varying particle size can change the retrieved $\tau$ by up to $2 \%$.

Cloud inhomogeneities may also affect the three retrieval methods in different ways. For case A, the time period selected for analysis reduced, but did not entirely eliminate, horizontal cloud inhomogeneities. For example, $\tau$ varied between 25 and 43 within $15 \mathrm{~min}$ around 15.4 UTC (Fig. 8). Cloud inhomogeneity has caused overestimations of cloud particle size for reflectance-based retrieval due to shadowing (Marshak et al., 2006). Shadowing may also cause an overestimate of cloud particle size for transmitted-radiance-based retrievals. Lastly, the presence of ice crystals may have been a cause of differences between the slope and two-wavelength methods in the work described by McBride et al. (2011). However, it is unlikely to be the cause of differences in this case. Ice crystal spectral absorption and scattering features are quantified with $\eta_{1}, \eta_{2}, \eta_{9}, \eta_{10}$, and $\eta_{13}$. If such features were observed, the cloud would be identified as an ice cloud.

\subsubsection{Modeled and measured radiance spectra comparison}

More insight into the differences between the three retrievals is gained when selecting a single sample measurement for further analysis. We compare a representative measurement spectrum to modeled spectra derived from the retrieved $\tau$ and $r_{\mathrm{e}}$ values from all three methods in Fig. 10. For all three cases, the root mean square (rms; see Table 2) difference throughout the entire wavelength range of the measured radiance spectra and the modeled radiance spectra based on the 15-parameter method are less than the other two methods by up to a factor of 3.8 smaller. However, radiances at the shortest wavelengths $(<1000 \mathrm{~nm})$ modeled using the slope method matched the measured spectra more closely than the 15-parameter method for the liquid cloud case (rms of $2.4 \%$ vs. $3.4 \%$ ) but not for the ice cloud case (rms of $7.7 \%$ vs. $4.9 \%)$.

For case A (Fig. 10), the fit of all modeled radiance spectra to the measured spectra is better than $24 \%$, resulting in rms values smaller than $6.4 \%$, with the largest variation from the measured spectra occurring at the longest wavelengths $(>1000 \mathrm{~nm}$ ). At wavelengths shorter than $1000 \mathrm{~nm}$, the mod- eled spectra based on all three retrievals matches the measured spectrum within $12 \%$. At $1500 \mathrm{~nm}$, the radiance difference for the modeled radiance spectra based on the slope retrieval diverges by $24 \%$. By contrast, the difference between measured and modeled radiance spectrum based on $\tau$ and $r_{\mathrm{e}}$ retrieved using the 15-parameter method is better than $7 \%$ at wavelengths longer than $1000 \mathrm{~nm}$ outside gas absorption bands and better than $8 \%$ at all wavelengths resulting in a rms of at least $2.8 \%$ lower than the other two methods. The better fit is expected for the 15-parameter method since the 15 parameters quantify spectral features located at wavelengths nearly spanning the entire range.

The sample measured spectrum for case B was selected to coincide at the transition between ice and liquid cloud, where both ice and liquid cloud particles influence the transmitted radiance spectrum. The retrieval described herein classified the cloud as liquid because the normalized radiance magnitudes indicated that the cloud was composed of liquid cloud droplets, although some ice particle absorption features are still apparent in the measured spectrum at wavelengths longer than $1000 \mathrm{~nm}$. One ice absorption feature is the monotonically decreasing radiance between 1000 and $1100 \mathrm{~nm}$, which is quantified by $\eta_{1}$ and $\eta_{9}$. Conversely, liquid cloud droplets produce a local maximum in radiance at $1050 \mathrm{~nm}$. We observed this maximum in the modeled radiance spectra based on the slope, two-wavelength, and 15parameter method (solid lines in Fig. 10). The ice absorption feature found in the measured radiance spectrum is reproduced in the dark green dashed line in Fig. 10, where there is no maximum at $1050 \mathrm{~nm}$. Between 1500 and $1700 \mathrm{~nm}$ the modeled ice cloud spectrum matches measurement more closely (within 16\%) than modeled liquid cloud spectrum based on the slope and two-wavelength methods (up to $28 \%$ difference), even though the smallest rms for the entire wavelength range $(7.2 \%)$ is obtained for the liquid cloud spectrum modeled from the 15-parameter method.

For case C (Fig. 10), the modeled radiance based on the 15-parameter method reproduced the measurement at wavelengths $>1000 \mathrm{~nm}$ more accurately than the modeled radiance based on the other two methods, similarly to case A. The modeled radiances based on the two-wavelength and slope method match nearly identically the measured radiance at $515 \mathrm{~nm}$ but diverge by up to $55 \%$ at wavelengths $>$ $1000 \mathrm{~nm}$. The poor fit of modeled radiance based on these two methods to measured radiance at longer wavelengths is likely due to the low signal-to-noise ratio of radiance at wavelengths near $1600 \mathrm{~nm}$, where ice absorption is largest. The best fit to the measured spectrum throughout the entire wavelength range is obtained by the modeled radiance spectrum based on the 15-parameter described in this work, with an rms of $5.9 \%$, which is 3.8 (3.4) times smaller than the rms from the slope (two-wavelength) method. This is expected since only 4 of the 15 spectral parameters rely on radiance with low signal-to-noise ratio, whereas half of the 

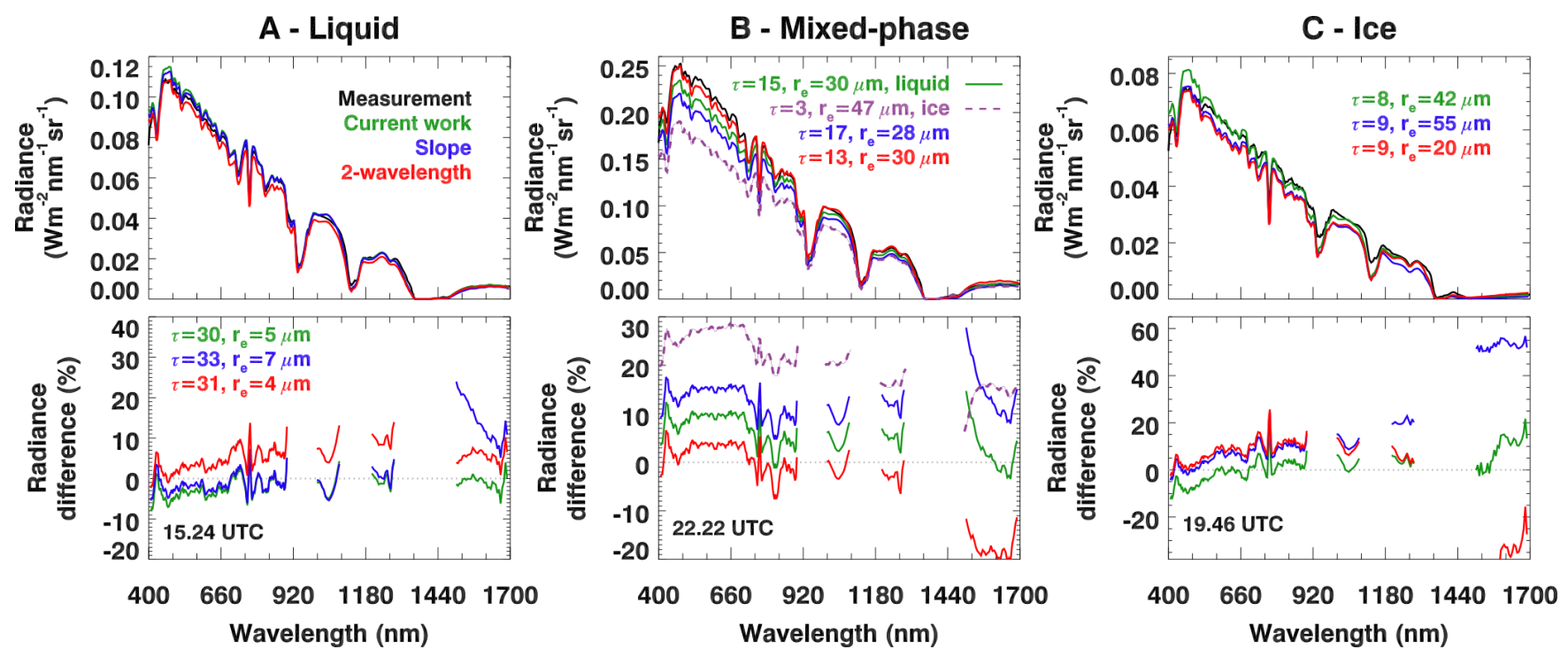

Figure 10. Sample measured and modeled spectra for the three different cloud case studies. Measured radiance spectra (black) are specific to a certain point (see legend) in the time series of Fig. 8. Modeled radiance spectra are based on the retrieved cloud properties from each method (this work is represented by green; slope is blue, and two-wavelength is red) for the same time instance. The percentage difference in the modeled spectra with respect to the measured spectra is shown in lower panels for wavelength regions outside of water vapor absorption bands. Modeled radiance spectra based on the current work's retrieval for an ice cloud (dashed purple line) and whether it was a liquid cloud (solid green line) is presented in case B. The slope and two-wavelength modeled spectra represent a liquid cloud in case B.

information for the slope and two-wavelength method rely on radiances at those wavelengths.

\section{Summary and conclusions}

This work introduces 15 new parameters quantifying unique absorption and scattering properties of ice and liquid water cloud particles and their first application to measured cloud radiance spectra to retrieve $\tau, r_{\mathrm{e}}$, and $\phi$. The 15 parameters generalize cloud retrieval techniques based on spectral radiance transmitted through clouds and were inspired by the spectral feature quantified by McBride et al. (2011). These parameters are derived from transmitted spectral radiance measurements which have been normalized by their maximum value or by the radiance value at $1000 \mathrm{~nm}$. By doing this, we no longer rely on the absolute radiometric calibration, which varies up to $8 \%$. Rather, we rely on the much higher wavelength-to-wavelength stability of the SSFR (4.6 or $1.1 \%$, depending on the normalization method). The measurements of spectral radiance were made by the SSFR based at Skywatch observatory in Boulder, Colorado, for three time periods encompassing clouds of different thermodynamic phase: liquid (case A), mixed-phase (case B), and ice (case $\mathrm{C}$ ). We retrieve $\tau, r_{\mathrm{e}}$, and $\phi$ by evaluating the weighted least-squares best fit between the parameters calculated from measured zenith radiance and precomputed tables of parameters derived from simulated cloud radiance. The new parameters are shown to distinguish cloud thermodynamic phase. This discrimination uses spectral absorption and scattering features unique to either ice or liquid water cloud particles. Thermodynamic phase discrimination for three cloud cases correctly reproduced the classification of the thermodynamic phase by ancillary data.

Five of the parameters, $\eta_{1}, \eta_{2}, \eta_{9}, \eta_{10}$, and $\eta_{13}$, give distinct values when either ice or liquid cloud particles are present. This sensitivity to thermodynamic phase is obtained by capitalizing on spectral differences in the absorption properties of ice and liquid cloud particles. Some of these parameters, $\eta_{9}$ and $\eta_{10}$ for example, are defined over spectral ranges where the position of the local maximum and minimum of transmittance differs for ice or liquid clouds. In those wavelength ranges, the transmitted normalized radiance for ice or liquid water clouds exhibit either a concave or convex shape, where the spectral slope increases or decreases, respectively, as wavelength increases.

The 15 parameters quantify specific spectral features found in normalized radiance. The normalization amplifies the spectral features of interest and allows for a more direct comparison of radiance spectra transmitted through different cloud, but it also removes the first-order dependence on $\tau$. After normalization, the parameters still show dependence on $\tau, r_{\mathrm{e}}$, and $\phi$. Of these 15 parameters, we have found that $\eta_{1}$, $\eta_{2}, \eta_{3}, \eta_{6}, \eta_{8}, \eta_{9}, \eta_{10}, \eta_{11}$, and $\eta_{15}\left(C_{1.0}, \frac{\partial}{\partial \lambda_{1.2}}, \frac{\partial}{\partial \lambda_{1.5}},\langle R\rangle_{1.6}\right.$, $C_{1.6}, \frac{\partial^{2}}{\partial \lambda_{1.0}^{2}}, \frac{\partial^{2}}{\partial \lambda_{1.2}^{2}}, \mathrm{sl}_{0.55}$, and $\mathrm{sl}_{1.6}$ ) show the largest sensitivity to $\tau, r_{\mathrm{e}}$, and $\phi$.

We compare $\tau$ and $r_{\mathrm{e}}$ from three different retrievals: the 15 -parameter retrieval described in this work, the spectral 
slope method described by McBride et al. (2011), and the two-wavelength method which is typically used with reflectance (Nakajima and King, 1990) but applied to transmittance (Kikuchi et al., 2006). For the liquid cloud case, the $\tau$ retrieved with the three different methods correlated well $\left(R^{2}=0.95\right)$ and matched within 10 , whereas $r_{\mathrm{e}}$ retrieved with the three methods did not match as well and correlated less (as low as $R^{2}=0.74$ ). One possible reason for the differences in retrieved $r_{\mathrm{e}}$ values can be attributed to the varying influence of cloud droplet vertical profile on radiance at different wavelength regions (Platnick, 2000).

We compared measured transmitted radiance spectra to modeled transmitted radiance spectra based on the retrieved $\tau, r_{\mathrm{e}}$, and $\phi$. We find root mean square differences between modeled and measured radiance spectra are less when using the results from the 15-parameter retrieval (3.1\%) than the slope and two-wavelength method by up to a factor of 3.8 for the ice cloud case. By contrast, differences in radiances approach $6.4 \%$ for the liquid case and $22.5 \%$ for the ice cloud case evaluated over the same wavelength range for modeled radiances based on slope and two-wavelength retrievals. At the shortest wavelengths (less than $1000 \mathrm{~nm}$ ), the slope method outperform this work's method for liquid clouds but not for ice clouds in terms of match to the measured spectra.
The use of spectral information in this work showcases the advantages of using a spectrometer system for remote sensing of clouds. Increased understanding of cloud properties can be gained using photons that have interacted with cloud particles throughout the entire cloud vertical extent. The basis of this method could also be applied to spectral reflectance measurements from satellites. Some of the spectral features investigated here may translate directly to reflectance, especially those based entirely on absorption properties of liquid and ice cloud particles. In future work, we will formally quantify uncertainties in the retrieved cloud properties from this new method and the information content of each parameter by investigating the impact of varying ancillary inputs on the retrieval scheme. This will be done using a nonlinear methodology (Vukicevic et al., 2010) that will investigate the changes of the retrieved properties over the full range of optical thickness and effective radius that results from uncertainties in the measurement and forward model inputs. 


\section{Appendix A: Description of case studies}

The SSFR was deployed at Skywatch from May 2012 to February 2013. From the collected data set, three cases were selected for further study based on criteria of (i) cloud horizontal homogeneity and (ii) variations in thermodynamic phase: liquid cloud (25 May 2012), mixed phase cloud (6 August 2012), and ice cloud (10 January 2013). In this section, we describe the ancillary data used to identify the case studies.

Cloud horizontal homogeneity is required for validity of the plane-parallel assumption in the forward model. We determined horizontal homogeneity by small temporal variability in cloud base height (measured from the ceilometer) and broadband downwelling irradiance (measured with the pyranometer for solar shortwave and pyrgeometer for thermal longwave). Visual inspection of time lapse movies, captured with a sky webcam over the selected time periods, was also used to filter for heterogeneous cloud cases. An example of data taken under heterogeneous and homogeneous cloud scenes is shown in Fig. A1. In this example, the heterogeneous case has variability in shortwave irradiance of more than $800 \mathrm{~W} \mathrm{~m}^{-2}$ over $10 \mathrm{~min}, 40 \mathrm{~W} \mathrm{~m}^{-2}$ in longwave irradiance over $20 \mathrm{~min}$, and cloud base heights varying from 0 (clear sky) to $6 \mathrm{~km}$. By contrast, the homogeneous cloud case has little variability in short- (less than $200 \mathrm{~W} \mathrm{~m}^{-2}$ over a $4 \mathrm{~h}$ period) and long-wave irradiance (less than $20 \mathrm{~W} \mathrm{~m}^{-2}$ over a $4 \mathrm{~h}$ period) and cloud base heights near $2 \mathrm{~km}$, varying by less than $1 \mathrm{~km}$.

The cloud thermodynamic phase was estimated using MRR, the ceilometer, atmospheric soundings, GOES, MODIS, and SSFR measurements of spectral radiance. The atmospheric soundings for the three different time periods are presented in Fig. A2. Cloud base height is retrieved using the ceilometer located at the Skywatch facility for the time of interest. The cloud top height was obtained from GOES for 25 May 2012 and 6 August 2012 and from MODIS for 10 January 2013. The GOES cloud top height for 25 May 2012 was below the freezing level obtained from the atmospheric sounding. Therefore, we assume that the cloud on 25 May 2012 was composed entirely of liquid water. The mixed phase case, 6 August 2012, and the ice case,
10 January 2013, had cloud base heights above the freezing level. On 6 August 2012, MRR measurements of backscatter indicated the presence of a melting layer that was also present $1 \mathrm{~h}$ prior to the selected case study period. In addition, GOES-retrieved cloud thermodynamic phase (Minnis et al., 1995), at cloud top, was ice $15 \mathrm{~min}$ prior to the measurement period and liquid during the measurement period. Therefore, we assume that the cloud on 6 August 2012 was likely mixed-phase, for this context meaning that both ice and liquid cloud particles influenced the transmitted radiation. On 10 January 2013 cloud base height was higher than $9.4 \mathrm{~km}$ above sea level, higher than the upper limit of the ceilometer measurement. During the measurement period on 10 January 2013, MODIS cloud phase was determined to be ice. SSFR measurements of zenith spectral radiance showed a sharp decrease in spectral radiance near 1000 and $1200 \mathrm{~nm}$ (not shown), representative of ice absorption features (Pilewskie and Twomey, 1987). Therefore, we assume that the cloud on 10 January 2013 was an ice cloud.

Surface albedos for the spring/summer measurement period were determined by scaling a measured, spectral albedo of a vegetated surface (Michalsky et al., 2003) (Fig. A3). This scaling matched the 16-day average surface albedo from MODIS for Boulder, Colorado, at the discrete spectral bands $470,555,645,858,1240,1640$, and $2130 \mathrm{~nm}$. We linearly interpolated between these bands and linearly extrapolated to shorter wavelengths to span the observed spectral range of SSFR. We applied this scaling to obtain the 16-day average surface albedo centered on 24 May 2012, on 4, 12, 20 August 2012, and on 13 September 2012, which spans our spring/summer measurement period (Fig. A3). We determined winter surface albedo (not shown) in a similar manner using a reference fresh snow surface albedo (Wiscombe and Warren, 1980) and scaling it to the retrieved albedo from MODIS for 10 January 2013 in the manner described above. The cloud base height was determined with the Skywatch ceilometer measurements and cloud top height from GOES and MODIS. 

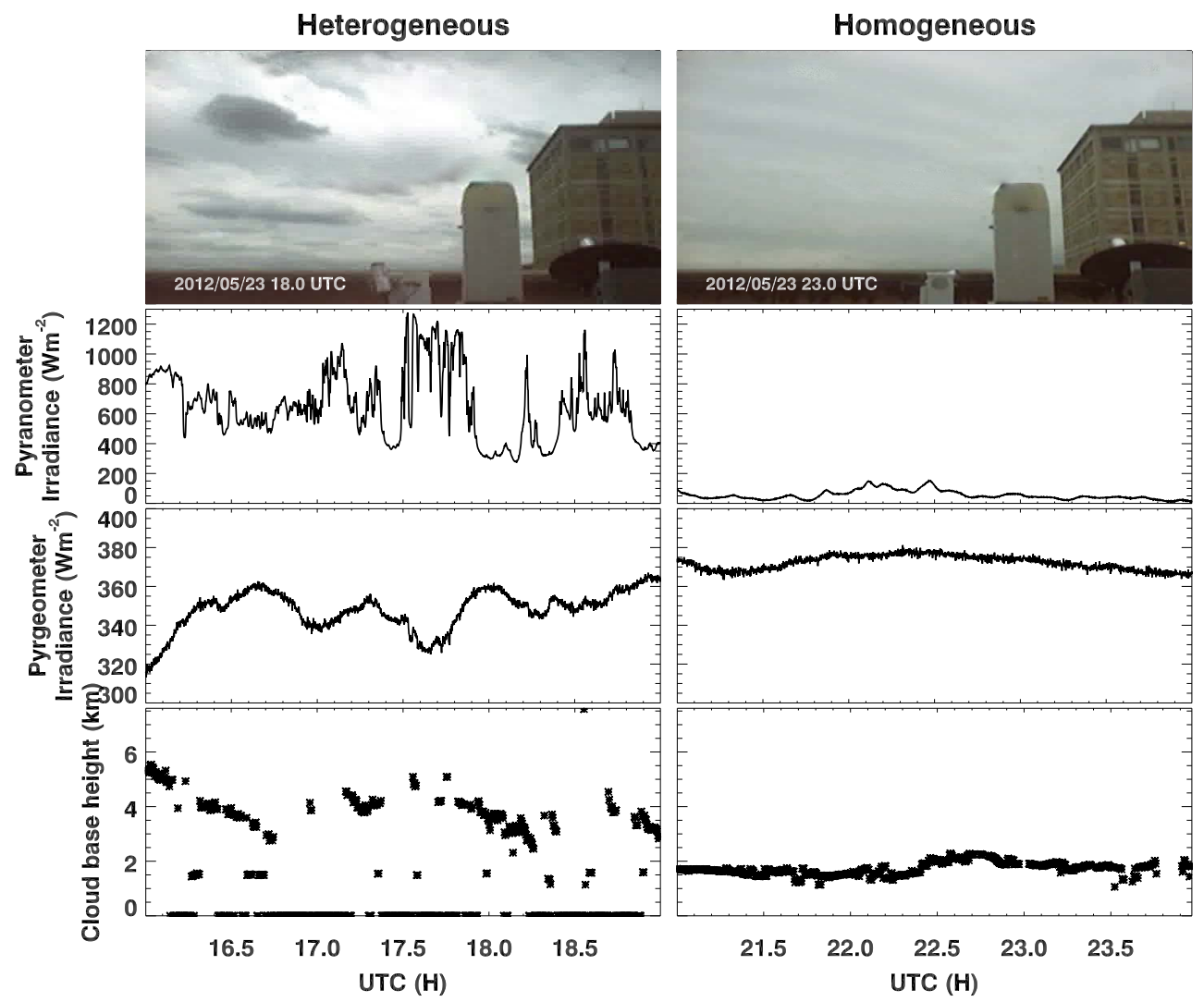

Figure A1. Broadband short- (pyranometer) and long-wave (pyrgeometer) irradiance and cloud base heights (ceilometer) for horizontally heterogeneous (left panel) and homogeneous (right panel) clouds taken on 23 May 2012. The top panel presents one image from the sky webcam for either heterogeneous or homogeneous clouds taken from the Skywatch observatory. 

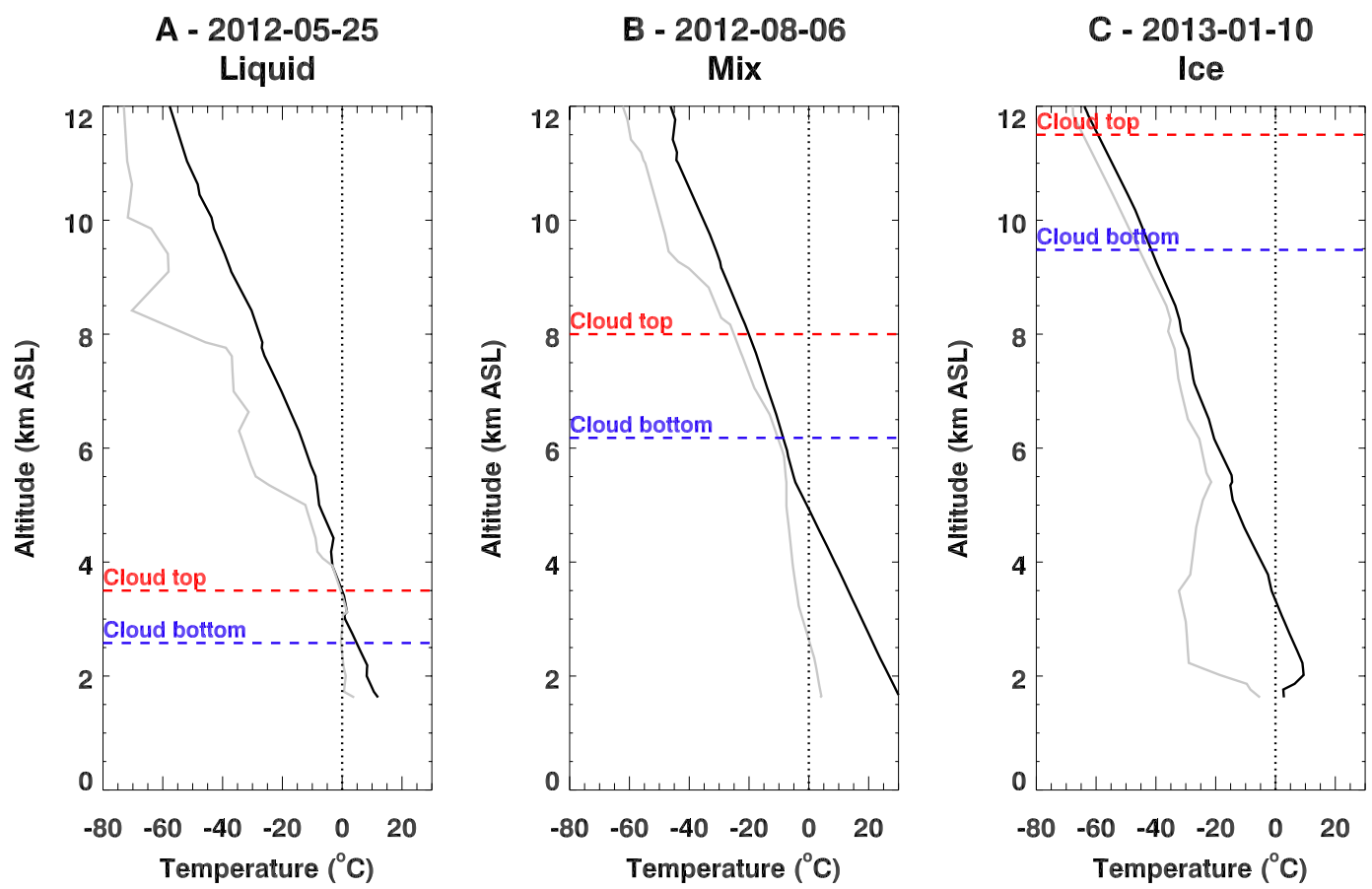

Figure A2. Atmospheric sounding profiles of temperature (black) and dew point (grey) taken near Denver International Airport for the case study time periods: (a) liquid cloud case on 25 May 2012, (b) mixed-phase cloud case on 6 August 2012, and (c) ice cloud case on 10 January 2013. Cloud bottom and top values determined from ground-based and satellite data (see text).

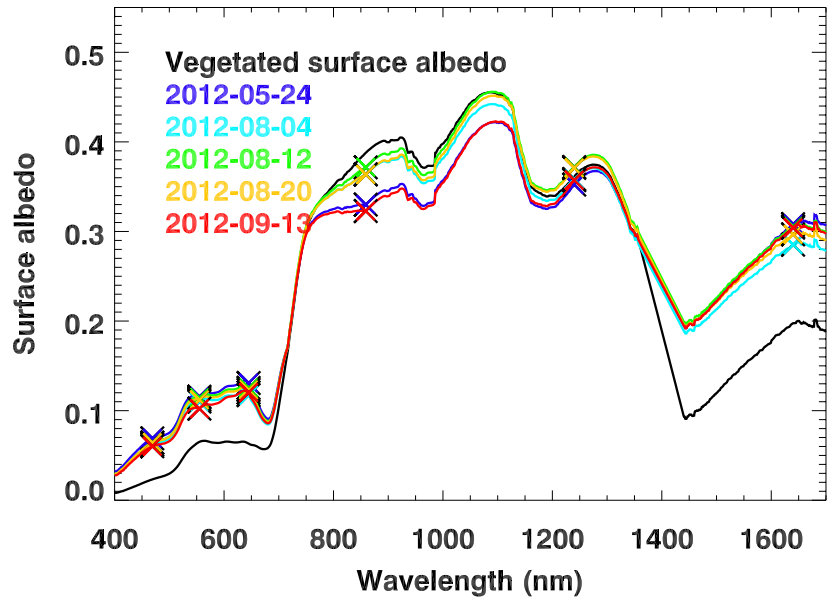

Figure A3. Spectral surface albedo used as input for the radiative transfer modeling for spring/summer. To approximate a full spectrum for varying days, the vegetated spectral surface albedo (black line) measured by Michalsky et al. (2003) is scaled using the discrete-band surface albedo product retrieved from Moderate Resolution Imaging Spectroradiometer (MODIS) measurements (Schaaf et al., 2002) at the wavelengths denoted by " $\mathrm{X}$ ". This scaling is interpolated between the discrete MODIS bands. 
Acknowledgements. This work was supported by NOAA award NA09OAR4310127 and NASA awards NNX11AE71G and NNX11AK67G. We thank Warren Gore from NASA Ames for the use of the SSFR instrument. The MODIS MCD43B3 surface albedo data were obtained through the online data pool at the NASA Land Processes Distributed Active Archive Center (LP DAAC), USGS/Earth Resources Observation and Science (EROS) Center, Sioux Falls, South Dakota (https://lpdaac.usgs.gov/get_data/).

Edited by: A. Kokhanovsky

\section{References}

Allan, R. P.: Combining satellite data and models to estimate cloud radiative effect at the surface and in the atmosphere, Meteorol. Appl., 18, 324-333, 2011.

Baum, B. A., Yang, P., Heymsfield, A. J., Schmitt, C. G., Xie, Y., Bansemer, A., Hu, Y.-X., and Zhang, Z.: Improvements in Shortwave Bulk Scattering and Absorption Models for the Remote Sensing of Ice Clouds, J. Appl. Meteorol. Climatol., 50, 10371056, doi:10.1175/2010JAMC2608.1, 2011.

Brückner, M., Pospichal, B., Macke, A. and Wendisch, M.: A new multispectral cloud retrieval method for ship-based solar transmissivity measurements, J. Geophys. Res.-Atmos., 119, 1-17, doi::10.1002/2014JD021775.Received, 2014.

Chiu, C., Marshak, A., Knyazikhin, Y., Wiscombe, W. J., Barker, H. W., Barnard, J. C., Luo, Y., and Chiu, J. C.: Remote sensing of cloud properties using ground-based measurements of zenith radiance, J. Geophys. Res., 111, D16201, doi:10.1029/2005JD006843, 2006.

Chiu, J. C., Huang, C.-H., Marshak, A., Slutsker, I., Giles, D. M., Holben, B. N., Knyazikhin, Y., and Wiscombe, W. J.: Cloud optical depth retrievals from the Aerosol Robotic Network (AERONET) cloud mode observations, J. Geophys. Res., 115, 1-12, doi:10.1029/2009JD013121, 2010.

Coddington, O. M., Pilewskie, P., Schmidt, K., McBride, P., and Vukicevic, T.: Characterizing a new surface-based shortwave cloud retrieval technique, based on transmitted radiance for soil and vegetated surface types, Atmosphere, 4, 48-71, doi:10.3390/atmos4010048, 2013.

Cumming, J. B.: Temperature dependence of light absorption by water, Nucl. Instruments Methods Phys. Res. Sect. A Accel. Spectrometers, Detect. Assoc. Equip., 713, 1-4, doi:10.1016/j.nima.2013.02.024, 2013.

Daniel, J. S.: Cloud liquid water and ice measurements from spectrally resolved near-infrared observations: a new technique, J. Geophys. Res., 107, 4599, doi:10.1029/2001JD000688, 2002.

Daniel, J. S., Solomon, S., Miller, H. L., Langford, A. O., Portmann, R. W., and Eubank, C. S.: Retrieving cloud information from passive measurements of solar radiation absorbed by molecular oxygen and $\mathrm{O}_{2}-\mathrm{O}_{2}$, J. Geophys. Res., 108, 1-12, doi:10.1029/2002JD002994, 2003.

Daniel, J. S., Portmann, R. W., Miller, H. L., Solomon, S., Langford, A. O., Eubank, C. S., Schofield, R., Turner, D. D., and Shupe, M. D.: Cloud property estimates from zenith spectral measurements of scattered sunlight between 0.9 and $1.7 \mu \mathrm{m}, \mathrm{J}$. Geophys. Res., 111, D16208, doi:10.1029/2005JD006641, 2006.
Evans, K. F.: The spherical harmonics discrete ordinate method for three-dimensional atmospheric radiative transfer, J. Atmos. Sci., 55, 429-446, doi:10.1175/15200469(1998)055<0429:TSHDOM>2.0.CO;2, 1998.

Hu, Y., Wielicki, B., Lin, B., Gibson, G., Tsay, S.-C., Stamnes, K., and Wong, T.: $\delta$-fit: a fast and accurate treatment of particle scattering phase functions with weighted singular-value decomposition least-squares fitting, J. Quant. Spectrosc. Ra., 65, 681-690, 2000.

Iwabuchi, H. and Hayasaka, T.: Effects of cloud horizontal inhomogeneity on the optical thickness retrieved from moderateresolution satellite data, J. Atmos. Sci., 59, 2227-2242, 2002.

Key, J. R. and Intrieri, J. M.: Cloud particle phase determination with the AVHRR, J. Appl. Meteorol., 39, 1797-1804, doi:10.1175/1520-0450-39.10.1797, 2000.

Kikuchi, N., Nakajima, T., Kumagai, H., Kuroiwa, H., Kamei, A., Nakamura, R., and Nakajima, T. Y.: Cloud optical thickness and effective particle radius derived from transmitted solar radiation measurements: comparison with cloud radar observations, J. Geophys. Res., 111, D07205, doi:10.1029/2005JD006363, 2006.

Kindel, B. C., Schmidt, K. S., Pilewskie, P., Baum, B. A., Yang, P., and Platnick, S.: Observations and modeling of ice cloud shortwave spectral albedo during the Tropical Composition, Cloud and Climate Coupling Experiment (TC 4), J. Geophys. Res., 115, D00J18, doi:10.1029/2009JD013127, 2010.

Kurucz, R. L.: Synthetic infrared-spectra, in: Infrared Solar Physics, edited by: Rabin, D. M., Jefferies, J. T., and Lindsey, C., no. 154 in IAU SYMPOSIA, Int. Astron. Union, Kluwer Academic Publ, P.O. Box 17, 3300 AA Dordrecht, the Netherlands, 523531, 1994.

LeBlanc, S. E., Schmidt, K. S., Pilewskie, P., Redemann, J., Hostetler, C., Ferrare, R., Hair, J., Langridge, J. M., and Lack, D. A.: Spectral aerosol direct radiative forcing from airborne radiative measurements during CalNex and ARCTAS, J. Geophys. Res., 117, D00V20, doi:10.1029/2012JD018106, 2012.

Marshak, A., Knyazikhin, Y., Evans, K. D., and Wiscombe, W. J.: The "RED versus NIR" plane to retrieve broken-cloud optical depth from ground-based measurements, J. Atmos. Sci., 61, 1911-1925, 2004.

Marshak, A., Platnick, S., Várnai, T., Wen, G., and Cahalan, R. F.: Impact of three-dimensional radiative effects on satellite retrievals of cloud droplet sizes, J. Geophys. Res., 111, D09207, doi:10.1029/2005JD006686, 2006.

Mayer, B. and Kylling, A.: Technical note: The libRadtran software package for radiative transfer calculations - description and examples of use, Atmos. Chem. Phys., 5, 1855-1877, doi:10.5194/acp-5-1855-2005, 2005.

McBride, P. J., Schmidt, K. S., Pilewskie, P., Kittelman, A. S., and Wolfe, D. E.: A spectral method for retrieving cloud optical thickness and effective radius from surface-based transmittance measurements, Atmos. Chem. Phys., 11, 7235-7252, doi:10.5194/acp-11-7235-2011, 2011.

McBride, P. J., Schmidt, K. S., Pilewskie, P., Walther, A., Heidinger, A. K., Wolfe, D. E., Fairall, C. W., and Lance, S.: CalNex cloud properties retrieved from a ship-based spectrometer and comparisons with satellite and aircraft retrieved cloud properties, J. Geophys. Res.-Atmos., 117, 1-10, doi:10.1029/2012JD017624, 2012. 
Michalsky, J., Min, Q., Barnard, J., Marchand, R., and Pilewskie, P.: Simultaneous spectral albedo measurements near the Atmospheric Radiation Measurement Southern Great Plains (ARM SGP) central facility, J. Geophys. Res., 108, 4254, doi:10.1029/2002JD002906, 2003.

Minnis, P., Kratz, D. P., Coakley Jr., J. A., King, M. D., Garber, D., Heck, P., Mayor, S., Young, D. F., and Arduini, R.: Cloud Optical Property Retrieval (Subsystem 4.3), Clouds and the Earth's Radiant Energy System (CERES) Algorithm Theoretical Basis Document, 3, 135-176, 1995.

Nakajima, T. and King, M. D.: Determinination of the optical thickness and effective particle radius of clouds from reflected solar radiation measurements. Part I: Theory, J. Atmos. Sci., 47, 1878-1893, doi:10.1175/15200469(1990)047<1878:DOTOTA>2.0.CO;2, 1990.

Nakajima, T. and Tanaka, M.: Algorithms for radiative intensity calculations in moderately thick atmospheres using a truncation approximation, J. Quant. Spectrosc. Ra., 40, 51-69, 1988.

Pilewskie, P. and Twomey, S.: Discrimination of ice from water in clouds by optical remote sensing, Atmos. Res., 21, 113-122, 1987.

Pilewskie, P., Pommier, J., Bergstrom, R., Gore, W., Howard, S., Rabbette, M., Schmid, B., Hobbs, P. V., and Tsay, S. C.: Solar spectral radiative forcing during the Southern African regional science initiative, J. Geophys. Res., 108, 1-7, doi:10.1029/2002JD002411, 2003.

Platnick, S.: Vertical photon transport in cloud remote sensing problems, J. Geophys. Res., 105, 22919-22935, 2000.

Platnick, S., Li, J. Y., King, M. D., Gerber, H., and Hobbs, P. V.: A solar reflectance method for retrieving the optical thickness and droplet size of liquid water clouds over snow and ice surfaces, J. Geophys. Res., 106, 15185-15199, 2001.

Platnick, S., King, M. D., Ackerman, S. A., Menzel, W. P., Baum, B. A., Riedi, J. C., and Frey, R. A.: The MODIS cloud products: algorithms and examples from Terra, IEEE T. Geosci. Remote, 41, 459-473, 2003.

Ptashnik, I. V., Smith, K. M., Shine, K. P., and Newnham, D. A.: Laboratory measurements of water vapour continuum absorption in spectral region $5000-5600 \mathrm{~cm}^{-1}$ : evidence for water dimers, Q. J. Roy. Meteorol. Soc., 130, 2391-2408, doi:10.1256/qj.03.178, 2004.

Rawlins, F. and Foot, J.: Remotely sensed measurements of stratocumulus properties during FIRE using the C130 aircraft multichannel radiometer, J. Atmos. Sci., 47, 2488-2504, 1990.

Ricchiazzi, P., Yang, S., Gautier, C., and Sowle, D.: SBDART: a research and teaching software tool for planeparallel radiative transfer in the Earth's atmosphere, B. Am. Meteorol. Soc., 79, 2101-2114, doi:10.1175/15200477(1998)079<2101:SARATS>2.0.CO;2, 1998.

Rothman, L. S., Gordon, I. E., Barbe, A., Benner, D. C., Bernath, P. F., Birk, M., Boudon, V., Brown, L. R., Campargue, A., and Champion, J. P.: The HITRAN 2008 molecular spectroscopic database, J. Quant. Spectrosc. Ra., 110, 533-572, doi:10.1016/j.jqsrt.2009.02.013, 2009.

Schaaf, C. B., Gao, F., Strahler, A. H., Lucht, W., Li, X., Tsang, T., Strugnell, N. C., Zhang, X., Jin, Y., Muller, J.-P., Lewis, P., Barnsley, M., Hobson, P., Disney, M., Roberts, G., Dunderdale, M., Doll, C., D’Entremont, R. P., Hu, B., Liang, S., Privette, J. L., and Roy, D.: First operational BRDF, albedo nadir reflectance products from MODIS, Remote Sens. Environ., 83, 135-148, doi:10.1016/S0034-4257(02)00091-3, 2002.

Schofield, R., Daniel, J. S., Portmann, R. W., Miller, H. L., Solomon, S., Eubank, C. S., Melamed, M. L., Langford, A. O., Shupe, M. D., and Turner, D. D.: Retrieval of effective radius and liquid water path from ground-based instruments: a case study at Barrow, Alaska, J. Geophys. Res., 112, D21203, doi:10.1029/2007JD008737, 2007.

Slingo, A.: Sensitivity of the Earth's radiation budget to changes in low clouds, Nature, 343, 49-51, 1990.

Stamnes, K., Tsay, S., Wiscombe, W. J., and Laszlo, I.: DISORT, a general-purpose Fortran program for discrete-ordinate-method radiative transfer in scattering and emitting layered media: documentation of methodology, Tech. rep., Dept. of Physics and Engineering Physic, Stevens Institute of Technology, Hoboken, NJ 07030, 2000.

Sun, Z. and Shine, K. P.: Studies of the radiative properties of ice and mixed-phase clouds, Q. J. Roy. Meteorol. Soc., 120, 111137, 1994.

Sun, Z. and Shine, K. P.: Parameterization of ice cloud radiative properties and its application to the potential climatic importance of mixed-phase clouds, J. Climate, 8, 1874-1888, 1995.

Taylor, J. R.: An introduction to error analysis: the study of uncertainties in physical measurements, University Science Books, 1997.

Turner, D. D., Vogelmann, A. M., Johnson, K., Miller, M., Austin, R. T., Barnard, J. C., Flynn, C., Long, C., McFarlane, S. A., Cady-Pereira, K., Clough, S. A., Chiu, J. C., Khaiyer, M. M., Liljegren, J., Lin, B., Minnis, P., Marshak, A., Matrosov, S. Y., Min, Q., O'Hirok, W., Wang, Z., and Wiscombe, W.: Thin liquid water clouds: their importance and our challenge, B. Am. Meteorol. Soc., 88, 177-190, doi:10.1175/BAMS-88-2-177, 2007.

Twomey, S. and Bohren, C. F.: Simple approximations for calculations of absorption in clouds, J. Atmos. Sci., 37, 2086-2095, doi:10.1175/1520-0469(1980)037<2086:SAFCOA>2.0.CO;2, 1980.

Twomey, S. and Cocks, T.: Remote sensing of cloud parameters from spectral reflectance in the near-infrared, Beiträge zur Physik der Atmosphäre, 62, 172-179, 1989.

Vukicevic, T., Coddington, O., and Pilewskie, P.: Characterizing the retrieval of cloud properties from optical remote sensing, J. Geophys. Res., 115, 1-14, doi:10.1029/2009JD012830, 2010.

Wan, Z. and Li, Z.-1.: A physics-based algorithm for retrieving land-surface emissivity and temperature from EOS/MODIS data, IEEE T. Geosci. Remote, 35, 980-996, 1997.

Warren, S. G. and Brandt, R. E.: Optical constants of ice from the ultraviolet to the microwave: a revised compilation, J. Geophys. Res., 113, 1-10, doi:10.1029/2007JD009744, 2008.

Wiscombe, W.: Improved Mie scattering algorithms, Appl. Optics, 19, 1505-1509, 1980.

Wiscombe, W. and Warren, S.: A model for the spectral albedo of snow. I: Pure snow, J. Atmos. Sci., 37, 2712-2733, doi:10.1175/1520-0469(1980)037<2712:AMFTSA>2.0.CO;2, 1980.

Wiscombe, W. J., Welch, R. M., and Hall, W. D.: The effects of very large drops on cloud absorption. Part I: Parcel models, J. Atmos. Sci., 41, 1336-1355, doi:10.1175/15200469(1984)041<1336:TEOVLD>2.0.CO;2, 1984. 
Zhang, S., Xue, H., and Feingold, G.: Vertical profiles of droplet effective radius in shallow convective clouds, Atmos. Chem. Phys., 11, 4633-4644, doi:10.5194/acp-11-4633-2011, 2011. 